\title{
GLOBAL SOLUTION FOR THE YANG-MILLS GRADIENT FLOW ON 4-MANIFOLDS
}

\author{
HIDEO KOZONO, YOSHIAKI MAEDA AND HISASHI NAITO ${ }^{\dagger}$
}

\section{Introduction}

In this paper, we will study a global weak solution for the Yang-Mills gradient flow on a closed (i.e., compact without boundary) 4-manifold. Let us explain some notion briefly to be able to state our results.

Let $M$ be a closed 4 -manifold, $G$ a compact Lie group embedded as a subgroup of $S O(l)$, or $S U(l)$ and $P$ be a principal $G$-bundle over $M$. We now assume the universal covering $\tilde{G}$ of $G$ is compact. Denote by $g$ the Lie algebra of $G$ and denote also by $\mathfrak{g}_{P}$ and $\mathscr{S}_{P}$ the adjoint and automorphism bundles of $P$, respectively. Using the metric on $G$ induced by the Killing form, we fix a metric on $P$ compatible with the action of $G$. Let $\Omega^{k}\left(\mathfrak{g}_{P}\right)$ be the space of smooth $\mathrm{g}$-valued $k$-forms, i.e., $\Omega^{k}\left(\mathfrak{g}_{p}\right)=C^{\infty}\left(M ; \mathfrak{g}_{P} \otimes \wedge^{k} T^{*} M\right)$. Here, for the space $\Omega^{k}\left(\mathfrak{g}_{p}\right)$ of $\mathfrak{g}_{P}$-valued $k$-forms, we can define Sobolev spaces $W^{m, p}, L^{p}$ with norms \|\|$_{W^{m, p} \text {, }}$ \|\|$_{p}$ in usual way.

Connections on $P$ are explained by taking an open covering $\left\{U_{\alpha}\right\}$ on $M$; we trivialize $P$ on $U_{\alpha}$ via a trivialization: $\left.P\right|_{U_{\alpha}} \cong U_{\alpha} \times G$. A connection $D$ on $P$ is, by definition, given by $D=d+A_{\alpha}$ on $U_{\alpha}$, where $A_{\alpha}$ is a g-valued 1 -form on $U_{\alpha}$. Moreover, for a set of transition functions $\left\{g_{\alpha \beta}\right\}$ of $P$ associated with the trivialization for $\left\{U_{\alpha}\right\}$, where $g_{\alpha \beta}: U_{\alpha} \cap U_{\beta} \rightarrow G, D$ satisfies

$$
A_{\beta}=g_{\alpha \beta}^{-1} d g_{\alpha \beta}+g_{\alpha \beta}^{-1} A_{\alpha} g_{\alpha \beta} \text { on } U_{\alpha} \cap U_{\beta} .
$$

We denote by $d_{D}$ and $d_{D}^{*}$ the covariant exterior differentiation and its formal adjoint with respect to a connection $D$, respectively. Moreover, the covariant differentiation on tensors for the connection $D$ is defined by $\tilde{\nabla}_{D}$. If $D$ is a smooth connection, then its curvature is given by $R_{D}=d_{D}{ }^{2} \in \Omega^{2}\left(\mathrm{~g}_{P}\right)$.

We consider the Yang-Mills gradient flow; the steepest descent flow of the

Received December 11, 1992.

Revised September 14, 1994.

${ }^{\dagger}$ Partially supported by the Ishida Foundation. 
Yang-Mills functional $E(D)=\frac{1}{2} \int_{M}\left|R_{D}\right|^{2} d V$ :

$$
\partial_{t} D=-d_{D}^{*} R_{D} \text { on } M \times[0, \infty),
$$

with the initial condition

$$
D(0)=D^{(0)} \text { on } M \times\{0\} .
$$

We will construct a global weak solution of (1.1), which may blow up in a finite time in the classical sense. If the solution blows up, then the structure of the bundle on which the connection lies may change. The notion of a weak of (1.1) is described as follows:

DEFINITION.

(1) The connection $D(t)$ is called a weak solution of (1.1) on the space $\left[T_{1}, T_{2}\right] \times M$ with the initial value $D\left(T_{1}\right)=D_{1}$ at $t=T_{1}$, if $D(t)$ is a connection on the same bundle $P$ for $t \in\left[T_{1}, T_{2}\right)$, and if the connection $D(t)$ satisfies

$$
\begin{aligned}
& \int_{T_{2}}^{T_{1}} \int_{M}\left\langle D, \partial_{t} \Phi\right\rangle-\left\langle R_{D}, d_{D} \Phi\right\rangle d V d t=\int_{M}\left\langle D_{1}, \Phi\left(T_{1}\right)\right\rangle d V, \\
& D(t) \in L^{2}\left(T_{1}, T_{2} ; W^{1,2}\left(\Omega^{1}\left(\mathfrak{g}_{P}\right)\right)\right), \\
& \sup _{T_{1}<t<T_{2}} \int_{M}\left|R_{D}(t)\right|^{2} d V<\infty,
\end{aligned}
$$

for any $\Phi \in C_{0}^{\infty}\left(\left[T_{1}, T_{2}\right) \times M, \Omega^{1}\left(g_{p}\right)\right)$, where the inner product $\langle D, \Phi\rangle$ for connection $D$ is defined by using the expression $D=D_{0}+A$ for fixed $D_{0}$.

(2) Moreover, a connection $D(t)$ is called a weak solution of (1.1) on $[0, \infty) \times M$ with the initial condition (1.2), if there exist finitely many collection of $G$-bundles $\left\{P_{i}\right\}_{i=1}^{L+1}$, where $P_{1}=P,\left\{t_{i}\right\}_{i=0}^{L+1}$, with $t_{0}=0, t_{L+1}$ $=\infty$, such that $D(t)$ is a weak solution on each $\left[t_{i}, t_{i+1}\right]$ with the initial value $D(0)=D^{(0)}$ at $t=0$ and $D\left(t_{i}\right)$ at $t=t_{i}(i=1, \ldots, L)$ in the above sense on the bundle $P_{i+1}$ and such that $R_{D}(t) \rightarrow R_{D}\left(t_{i+1}\right)$ weakly in $L^{2}(M)$ as $t \uparrow t_{i+1}$.

Let $\tilde{G}$ be the universal covering space of $G$ and let $K=\pi^{-1}(e)$, where $e$ is the identity element of $G$. Let $\tilde{g}_{\alpha \beta}$ be a lift of transition functions $g_{\alpha \beta}$ on $\tilde{G}$. Since $g_{\alpha \beta} \cdot g_{\beta \gamma} \cdot g_{\gamma \alpha}=e$, 


$$
f_{\alpha \beta \gamma}: U_{\alpha} \cap U_{\beta} \cap U_{\gamma} \rightarrow K, \quad f_{\alpha \beta \gamma}=\tilde{g}_{\alpha \beta} \cdot \tilde{g}_{\beta \gamma} \cdot \tilde{g}_{\gamma \alpha}
$$

is well-defined and satisfies $\pi f_{\alpha \beta \gamma}=e,\left\{f_{\alpha \beta \gamma}\right\}$ determines the element of $H^{2}(M, K)$. We denote this element by $\eta(P)$ (cf. [8]).

The first purpose of this paper is to show the following:

Theorem A. For any $D^{(0)} \in \mathfrak{u}^{1,2}$, there exists a weak global solution $D(t)$ of (1.1) with the initial condition (1.2) on $[0, \infty) \times M$. More precisely, we have the following:

(i) There exist finite set of times $\left\{t_{i}\right\}_{l=0}^{L+1}$, with $t_{0}=0$ and $t_{L+1}=\infty$, and $G$-bundles $\left\{P_{i}\right\}_{i=1}^{L}$ with $P_{1}=P$ such that $D(t)$ is a smooth connection of $P_{i}$ on $\left(t_{i}, t_{i+1}\right)$ and satisfies $(1.1)$ in the classical sense.

(ii) For each $i$, there exist $N_{i}(<\infty)$ points $\left\{x_{i_{j}}\right\}_{i=1}^{N_{i}}$ of $M$ such that $D\left(t_{i}\right)$ is a connection of $\left.P_{i}\right|_{M \backslash \cup_{j=1}^{N_{i}}\left\{x_{i}\right\}}$.

(iii) The function $t \mapsto \int_{M}\left|R_{D}(t)\right|^{2} d V$ is non-increasing and weakly continuous in $L^{2}(0, \infty)$.

(iv) Each bundle $P_{i}$ satisfies $\eta\left(P_{i}\right)=\eta(P)$.

Here, the space $\mathfrak{u}^{m, p}$ is defined by as follows. Fix an open covering $\left\{U_{\alpha}\right\}$ of $M$ which trivialize $P$ on $U_{\alpha}$. Now, the connection $D$ is expressed by $d+A_{\alpha}$ on $U_{\alpha}$, so we define

$$
\mathfrak{u}^{m, p}=\left\{D=d+A_{\alpha}:\left\|A_{\alpha}\right\|_{W^{m, p}}<\infty\right\},
$$

where \|\|$_{W^{m, p}}$ denotes the $W^{m, p}$-norm given by the trivialization $\left.P\right|_{U_{\alpha}} \cong U_{\alpha} \times G$.

The weak global solution $D(t)$ of (1.1) as described in Theorem A should be viewed as leading in the first step from the initial connection $D\left(t_{0}\right)=D(0)$ to the ideal connection $D\left(t_{1}\right)$ on $P=P_{1}$, a point of the boundary of the space of connections, and their within that boundary, to a new ideal connection $D\left(t_{2}\right)$ on a modified bundle $P_{2}$. It will be proved that in a finite number of such steps the solution can be extended to the interval $[0, \infty)$. The procedure is very much in accordance with the structure of the module space compactification as elucidated by Donaldson and Uhlenbeck.

The gauge transformation $s \in \mathscr{B}=C^{\infty}\left(\mathscr{S}_{P}\right)$ acts on connections: $A_{\alpha} \mapsto s_{\alpha}^{*} A_{\alpha}$ $=s_{\alpha}^{-1} d s_{\alpha}+s_{\alpha}^{-1} A_{\alpha} s_{\alpha}$. The curvature is actually a section of the bundle $P \otimes T^{*} M$ $\wedge T^{*} M$, and so a gauge transformation $s \in \mathbb{B}$ also acts on curvature tensors by $R_{D} \mapsto s^{*} R_{D}=R_{s^{*} D}=s^{-1} R_{D} s$. Note that gauge transformations leave the Yang-Mills functional invariant i.e., $E\left(s^{*} D\right)=E(D)$. This is a crucial difficulty 
for treating the smooth solution of (1.1). At first we construct a solution of (1.1) in a finite interval $(0, T)$ by using the following trick: If a connection $D$ transforms to $s^{*} D=\tilde{D}$ under a gauge transformation $s$, then the equation of the Yang-Mills gradient flow (1.1) is transformed to

$$
\begin{cases}\partial_{t} D=-d_{D}^{*} R_{D}+d_{D} \alpha & \text { on } M \times[0, \infty), \\ D(0)=D^{(0)} & \text { on } M \times\{0\},\end{cases}
$$

where $\alpha=s^{-1} \partial_{t} s \in \Omega^{0}\left(\mathfrak{g}_{P}\right)$ (cf. Jost [2]). We call (1.3) a modified Yang-Mills gradient flow. Conversely, a solution $D, \alpha$ or $s$ of (1.3) yields a solution $\left(s^{-1}\right)^{*} D$ of (1.1).

To obtain Theorem A, we constructed a solution of (1.3) in a finite time interval $(0, T)$, and return to (1.1). We also show that the energy functional $E(D(t))$ is monotone non-increasing with respect to $t$. Such a monotonicity of the energy functional can extend the life span of our local solution beyond $T$. The singular set $\&$ can be characterized in terms of the local concentration of the $L^{2}$-norm of the curvature $R_{D}$.

THEOREM B. The singular set $\&=\left\{\left(x_{i}, t_{i}\right) \in M \times(0, \infty], i=1, \ldots, L+1\right\}$ for the weak solution given in Theorem $\mathrm{A}$ is characterized as follows: There exists a positive constant $\varepsilon_{1}$ depending only on $M$ and $G$ such that

$$
\limsup _{t \uparrow t_{i}} \int_{B_{r}\left(x_{i j}\right)}\left|R_{D}(t)\right|^{2} d V \geq \varepsilon_{1}
$$

for all $r>0$.

For a principal bundle $P$ on $M$, we take a connection $D_{0}$ and fix it. Any connection $D$ is expressed as $D=D_{0}+A$ where $A \in \Omega^{1}\left(\mathrm{~g}_{P}\right)$.

Our second purpose is to discuss the uniqueness of weak solutions to (1.1). To this end, let us introduce a class $X(M,(0, T))$ of connections:

$$
X(M, 0, T))=\left\{D(t) \in \mathfrak{U}^{1,2}: \sup _{0<t<T} \int_{M}\left(\left|R_{D}\right|^{2}+\left|\tilde{\nabla}_{D} R_{D}\right|^{2}+\left|\tilde{\nabla}_{D}^{2} R_{D}\right|^{2}\right) d V<\infty\right\}
$$

Theorem C. Let $D=D_{0}+A$ and $\bar{D}=D_{0}+\bar{A}$ be two weak solutions of (1.1) with the initial condition (1.2), $D^{(0)} \in \mathfrak{u}^{1,2}$ in the class of $X(M,(0, T))$. Suppose in addition that $A, \bar{A} \in L^{q}\left(0, T ; L^{r}\left(\Omega^{1}\left(g_{p}\right)\right)\right)$ for $q \geq 2$ and $r>4$ with $2 / q+4 / r$ $\leq 1$. If $d_{D_{0}}^{*} A, d_{D_{0}}^{*} \bar{A} \in W^{1, \infty}\left(M \times[0, T) ; \Omega^{0}\left(\mathfrak{g}_{P}\right)\right)$, then there exist gauge transformations $s$ and $\bar{s}$ in the class $W^{1, \infty}\left(M \times[0, T) ; \mathbb{S}_{P}\right)$ such that $s^{*} A=\bar{s}{ }^{*} \bar{A}$ on 
$M \times[0, T)$.

\section{Fundamental inequalities}

In this section, we give some fundamental inequalities for later use.

PROPOSITION 2.1. There exist constants $C, R_{0}>0$ such that for any $u \in$ $W^{1,2}(M), v \in W^{1,2}(M)$, and any $r \in\left(0, R_{0}\right]$, we have

$$
\int_{M}|u||v|^{2} d V \leq C \sup _{x \in M}\left(\int_{B_{\gamma}(x)}|u|^{2} d V\right)^{1 / 2}\left(\int_{M}|\nabla v|^{2} d V+r^{-2} \int_{M}|v|^{2} d V\right) .
$$

We first show a local version to Proposition 2.1.

Lemma 2.2. There exist constants $C, R_{0}>0$ such that for any $u \in W^{1,2}(M)$, $v \in W^{1,2}(M), r \in\left(0, R_{0}\right], x \in M$ and a monotone non-increasing non-negative radial function $\varphi=\varphi(\operatorname{dist}(x, \cdot)) \in L^{\infty}(M)$ with $\varphi \equiv 0$ on $M \backslash B_{r}(x)$, the following inequality holds:

$$
\int_{M}|u||v|^{2} \varphi d V \leq C\left(\int_{B_{r}(x)}|u|^{2} d V\right)^{1 / 2}\left(\int_{M}|\nabla v|^{2} \varphi d V+r^{-2} \int_{M}|v|^{2} \varphi d V\right) .
$$

Proof. First we assume $\varphi=1$ on $B_{r}(x)$ and let $\bar{v}=\operatorname{vol}\left(B_{r}(x)\right)^{-1} \int_{B_{r}(x)} v d V$ be the mean value of $v$ on $B_{r}(x)$. By Hölder's inequality, we have

$$
\begin{aligned}
\int_{B_{r}}|u||v|^{2} d V & \leq C\left(\int_{B_{r}}|u|^{2} d V\right)^{1 / 2}\left(\int_{B_{r}}|v|^{4} d V\right)^{1 / 2} \\
& \leq C\left(\int_{B_{r}}|u|^{2} d V\right)^{1 / 2}\left(\int_{B_{r}}|v-\bar{v}|^{4}+|\bar{v}|^{4} d V\right)^{1 / 2} .
\end{aligned}
$$

By the Sobolev embedding theorem, we have

$$
\int_{B_{r}}|v-\bar{v}|^{4} d V \leq C\left(\int_{B_{r}}|\nabla v|^{2} d V\right)^{2} .
$$

On the other hand, by Hölder's inequality,

$$
\begin{aligned}
\int_{B_{r}}|\bar{v}|^{4} d V & \leq C \int_{B_{r}}\left|\frac{1}{\operatorname{vol}\left(B_{r}\right)} \int_{B_{r}} v d V\right|^{4} d V \\
& \leq C \operatorname{vol}\left(B_{r}\right)^{-3}\left|\int_{B_{r}} v d V\right|^{4}
\end{aligned}
$$




$$
\begin{aligned}
& \leq C \operatorname{vol}\left(B_{r}\right)^{-1}\left(\int_{B_{r}}|v|^{2} d V\right)^{2} \\
& \leq C r^{-4}\left(\int_{B_{r}}|v|^{2} d V\right)^{2} .
\end{aligned}
$$

By (2.1), (2.2) and (2.3), we get Lemma 2.2 for $\varphi=1$.

By linearity, Lemma 2.2 holds also for step functions. For general $\varphi$, we can show the assertion by approximating $\varphi$ in measure by step functions.

Proposition 2.1 is derived from Lemma 2.2 via the following lemma. For the proof, see Struwe [9].

LEMma 2.3. There exist constants $K, R_{0}>0$ depending only on $M$ such that for any $r \in\left(0, R_{0}\right]$ there exists a covering of $M$ by balls $B_{r / 2}\left(x_{i}\right)$ satisfying that at any point $x \in M$ at most $K$ of the balls $B_{r}\left(x_{i}\right)$ meet.

Next, we give identities for the curvature form $R_{D}$ for a connection $D$ :

LEMma 2.4. If $D$ is a smooth solution of (1.1), then

$$
\begin{aligned}
& \partial_{t} R_{D}=-\Delta_{D}^{H} R_{D}, \\
& \partial_{t} R_{D}=-\Delta_{D}^{r} R_{D}+\left[R_{D}, R_{D}\right], \\
& \partial_{t}\left|R_{D}\right| \leq \Delta\left|R_{D}\right|+C\left|R_{D}\right|^{2} \\
& \partial_{t}\left|\tilde{\nabla}_{D}^{(n)} R_{D}\right| \leq \Delta\left|\tilde{\nabla}_{D}^{(n)} R_{D}\right|+C \sum_{i=0}^{n}\left|\tilde{\nabla}_{D}^{(i)} R_{D}\right|\left|\tilde{\nabla}_{D}^{(n-i)} R_{D}\right|, \text { for } n=1,2, \ldots
\end{aligned}
$$

where $\tilde{\nabla}_{D}^{(i)}$ denotes the covariant differentiation of $i$-th order with respect to $\tilde{\nabla}_{D}$, and $\Delta_{D}^{H}$ and $\Delta_{D}^{r}$ are the Hodge and the rough Laplacian, respectively, i.e., $\Delta_{D}^{H}=d_{D}^{*} d_{D}+d_{D} d_{D}^{*}$ and $\Delta_{D}^{r}=D^{*} D$.

Proof. Note that $d_{D} \partial_{t} D=\partial_{t} R_{D}$. Applying $d_{D}$ to (1.1), we have, by the Bianchi indentity,

$$
\partial_{t} R_{D}=-d_{D} d_{D}^{*} R_{D}=-\Delta_{D}^{H} R_{D}
$$

The Bochner-Weizenböck formula gives

$$
\left(\Delta_{D}^{r}-\Delta_{D}^{H}\right) R_{D}=\left[R_{D}, R_{D}\right],
$$

hence we obtain (2.4) and (2.5). 
Moreover, for $\phi \in \Omega^{2}\left(\mathfrak{g}_{P}\right)$ we have

$$
|\phi| \Delta|\psi| \geq\left\langle\phi,-\Delta_{D}^{r} \psi\right\rangle
$$

which implies (2.6).

To obtain the inequality (2.7), we may show

$$
\partial_{t} \tilde{\nabla}_{D}^{(n)} R_{D}=\tilde{\nabla}_{D}^{2} \tilde{\nabla}_{D}^{(n)} R_{D}+\sum_{i=0}^{n} \tilde{\nabla}_{D}^{(i)} R_{D} * \tilde{\nabla}_{D}^{(n-i)} R_{D}
$$

where $A * B$ denotes some linear combination of tensor products of components of $A$ and $B$. Indeed, the case $n=0$ is obtained by (2.5). Assuming (2.8) for $n$ and using (1.1), we have

$$
\begin{aligned}
\partial_{t} \tilde{\nabla}_{D} \tilde{\nabla}_{D}^{(n)} R_{D} & =\tilde{\nabla}_{D} \partial_{t} \tilde{\nabla}_{D}^{(n)} R_{D}+\left[d_{D}^{*} R_{D}, \tilde{\nabla}_{D}^{(n)} R_{D}\right] \\
& =\tilde{\nabla}_{D}\left(\tilde{\nabla}_{D}^{2} \tilde{\nabla}_{D}^{(n)} R_{D}+\sum_{i=0}^{n} \tilde{\nabla}_{D}^{(i)} R_{D} * \tilde{\nabla}_{D}^{(n-i)} R_{D}\right)+\left[d_{D}^{*} R_{D}, \tilde{\nabla}_{D}^{(n)} R_{D}\right] \\
& =\tilde{\nabla}_{D}^{2}\left(\tilde{\nabla}_{D} \tilde{\nabla}_{D}^{(n)} R_{D}\right)+\sum_{i=0}^{n} \tilde{\nabla}_{D}^{(i)} R_{D} * \tilde{\nabla}_{D}^{(n+i-i)} R_{D}
\end{aligned}
$$

which implies (2.8) for $n+1$.

\section{Construction of the local strong solution}

In this section, we show the existence of a time-local smooth solution for (1.3). First we rewrite (1.3) as an equation for the connection $D=D_{0}+A$, where $D_{0}$ is a fixed connection on $P$. To make (1.3) a parabolic system for $A$, we take $\alpha=-d_{A}^{*} A$, (cf. Kono-Nagasawa [3]). Taking $\nabla=D_{0}$, we see that (1.3) is equivalent to the following equations (cf. Naito-Kozono-Maeda [7]):

$$
\left\{\begin{aligned}
\frac{\partial A_{i}(t)}{\partial t} & =\nabla^{j} \nabla_{j} A_{i}-\left[R_{i}^{j}, A_{j}\right]-d_{D_{0}}^{*} R \\
& +\left[A^{j}, \nabla_{j} A_{i}+\left[A_{j}, A_{i}\right]\right]+\left[\nabla_{i} A^{j}-\nabla^{j} A_{i}+\left[A_{i}, A^{j}\right], A_{j}\right] \\
& +\nabla^{j}\left[A_{j}, A_{i}\right]+\left[A^{j},\left[A_{j}, A_{i}\right]\right], \\
\left.A_{i}(t)\right|_{t=0} & =A_{i}^{(0)},
\end{aligned}\right.
$$

where $A(t)=A_{i}(t) d x^{i} \in \Omega^{1}\left(\mathfrak{g}_{p}\right)$ is the unknown function, $A^{(0)}=A_{i}^{(0)} d x^{i} \in$ $\Omega^{1}\left(\mathrm{~g}_{P}\right)$ is the given initial data, and $R=R_{i j} d x^{i} \wedge d x^{j}$ is the curvature 2 -form of $D_{0}$.

For the construction of the local solution for (3.1), we do not need to restrict the dimension of $M$. Making use of fractional powers of the Laplacian, we shall 
prove the existence of a strong solution $A(t)$ of (3.1) on a finite time interval $(0, T)$.

Let us introduce some notations: The space $L^{r}\left(\Omega^{1}\left(\mathfrak{g}_{p}\right)\right)$ denotes the usual $L^{r}$-space with the norm \|\|$_{r}$. We define an operator $\mathscr{L}_{r}$ on $L^{r}\left(\Omega^{1}\left(\mathfrak{g}_{P}\right)\right)$ by

$$
\mathscr{L}_{r} A_{i}:=-\nabla^{j} \nabla_{j} A_{i}+\left[R_{i}^{j}, A_{j}\right] \text {, for } A \in D\left(\mathscr{L}_{r}\right)
$$

with the domain $D\left(\mathscr{L}_{r}\right)=W^{2, r}\left(\Omega^{1}\left(\mathfrak{g}_{p}\right)\right)$.

(3.1) may be rewritten as the following equation on $L^{r}\left(\Omega^{1}\left(g_{P}\right)\right)$ :

$$
\left\{\begin{array}{l}
\frac{\partial A}{\partial t}+\mathscr{L}_{r} A+Q(A)=-d_{D_{0}}^{*} R, \\
A(0)=A^{(0)},
\end{array}\right.
$$

where $Q(A)=Q_{1}(A)+Q_{2}(A)$;

$$
\begin{aligned}
& Q_{1}(A)_{i}=-2\left[A^{j}, \nabla_{j} A_{i}\right]-\left[\nabla_{i} A^{j}, A_{j}\right]-\nabla^{j}\left[A_{J}, A_{i}\right], \\
& Q_{2}(A)_{i}=-3\left[A^{j},\left[A_{j}, A_{i}\right]\right] .
\end{aligned}
$$

Our result now reads as follows.

Theorem 3.1. Let $\operatorname{dim} M=n$ and let $A^{(0)} \in L^{n}\left(\Omega^{1}\left(\mathfrak{g}_{p}\right)\right)$. Then there exist $T>$ 0 and a function $A(t)$ on $[0, T)$ with the following properties:

(1) $A \in C\left([0, T) ; L^{n}\left(\Omega^{1}\left(\mathfrak{g}_{P}\right)\right)\right) \cap C^{1}\left((0, T) ; L^{n}\left(\Omega^{1}\left(\mathfrak{g}_{P}\right)\right)\right)$;

(2) $A(t) \in D\left(\mathscr{L}_{n}\right)$ for $t>0, \mathscr{L}_{n} A \in C\left((0, T) ; L^{n}\left(\Omega^{1}\left(g_{P}\right)\right)\right)$;

(3) $A$ is a solution of (3.2).

In this section, we are interested only in constructing the local solution of (3.1). Changing the unknown connection $A(t)$ into $A^{\prime}(t)$ by the relation $A^{\prime}(t)=$ $e^{\lambda t} A(t)$, we may assume that $\mathscr{L}_{r}$ has a bounded inverse $\mathscr{L}_{r}^{-1}$ on $L^{r}\left(\Omega^{1}\left(\mathrm{~g}_{P}\right)\right)$, where $\lambda$ is a constant larger than the smallest eigenvalue of $\mathscr{L}_{r}$. To prove Theorem 3.1, we need some lemmas. By the well-known theory of elliptic differential equations,

$$
\|A\|_{H^{2, r}} \leq C_{r}\left\|\mathscr{L}_{r} A\right\|_{r}, \quad \text { for } \quad A \in D\left(\mathscr{L}_{r}\right) \quad(1<r<\infty)
$$

with a constant $C_{r}$ independent of $A$. Moreover, $-\mathscr{L}_{r}$ generates a contractive holomorphic semigroup $\left\{e^{-t \mathscr{Q}_{r}}\right\}_{t \geq 0}$ of class $C^{0}$ in $L^{r}\left(\Omega^{1}\left(g_{P}\right)\right)$. Therefore, we can define the fractional power $\mathscr{L}_{r}^{\alpha}(0<\alpha<1)$ of $\mathscr{L}_{r}$ and get a continuous embedding

$$
D\left(\mathscr{L}_{r}^{\alpha}\right) \hookrightarrow H^{2 \alpha, r}\left(\Omega^{1}\left(\mathfrak{g}_{p}\right)\right), \quad 0 \leq \alpha \leq 1,
$$

where $H^{m, r}$ denotes the space of the Bessel potentials. (see, e.g., Fujiwara [1]). 
In the following, we shall work mainly with $r=n$ and write $\mathscr{L}_{n}=\mathscr{L}$ for simplicity.

Lemma 3.2. If $A \in D\left(\mathscr{L}^{\alpha}\right)$ for $\frac{1}{2}<\alpha<1$, then $Q_{1}(A), Q_{2}(A) \in L^{n}\left(\Omega^{1}\left(\mathfrak{g}_{P}\right)\right)$. In fact,

$$
\begin{aligned}
& \left\|Q_{1}(A)\right\|_{n} \leq C\left\|\mathscr{L}^{\alpha} A\right\|_{n}\left\|\mathscr{L}^{1 / 2} A\right\|_{n}, \\
& \left\|Q_{2}(A)\right\|_{n} \leq C\left\|\mathscr{L}^{\alpha} A\right\|_{n}\left\|\mathscr{L}^{1 / 4} A\right\|_{n}^{2} .
\end{aligned}
$$

If $A, B \in D\left(\mathscr{L}^{\alpha}\right)$ for $\frac{1}{2}<\alpha<1$, then

$$
\begin{aligned}
\left\|Q_{1}(A)-Q_{1}(B)\right\|_{n} & \leq C\left(\left\|\mathscr{L}^{\alpha}(A-B)\right\|_{n}\left\|\mathscr{L}^{1 / 2} B\right\|_{n}\right. \\
& \left.+\left\|\mathscr{L}^{\alpha} A\right\|_{n}\left\|\mathscr{L}^{1 / 2}(A-B)\right\|_{n}\right), \\
\left\|Q_{2}(A)-Q_{2}(B)\right\|_{n} & \leq C\left(\left\|\mathscr{L}^{1 / 4} A\right\|_{n}^{2}+\left\|\mathscr{L}^{1 / 4} B\right\|_{n}^{2}\right)\left\|\mathscr{L}^{\alpha}(A-B)\right\|_{n},
\end{aligned}
$$

where the constant $C$ depends only on $\alpha$.

Proof. By (3.4) and the Sobolev embedding, we have $D\left(\mathscr{L}^{\alpha}\right) \hookrightarrow L^{\infty}\left(\Omega^{1}\left(\mathrm{~g}_{p}\right)\right)$, $D\left(\mathscr{L}^{1 / 2}\right) \hookrightarrow H^{1, n}\left(\Omega^{1}\left(\mathrm{~g}_{P}\right)\right)$ and $D\left(\mathscr{L}^{1 / 4}\right) \hookrightarrow L^{2 n}\left(\Omega^{1}\left(\mathfrak{g}_{P}\right)\right)$, where $\hookrightarrow$ means a continuous inclusion. Hence it follows from Hölder's inequality that

$$
\begin{aligned}
& \left\|Q_{1}(A)\right\|_{n} \leq C\|A\|_{\infty}\|\nabla A\|_{n} \leq C\left\|\mathscr{L}^{\alpha} A\right\|_{n}\left\|\mathscr{L}^{1 / 2} A\right\|_{n}, \\
& \left\|Q_{2}(A)\right\|_{n} \leq C\|A\|_{2 n}^{2}\|A\|_{\infty} \leq C\left\|\mathscr{L}^{\alpha} A\right\|_{n}\left\|\mathscr{L}^{1 / 4} A\right\|_{n}^{2},
\end{aligned}
$$

which shows (3.5). The inequality (3.6) is an immediate consequence of (3.5).

LEMMA 3.3.

If $A \in D\left(\mathscr{L}^{\alpha}\right)$ for $\frac{1}{2}<\alpha<1$, then

$$
\begin{aligned}
& \left\|\mathscr{L}^{-1 / 4} Q_{1}(A)\right\|_{n} \leq M\left\|\mathscr{L}^{1 / 4} A\right\|_{n}\left\|\mathscr{L}^{1 / 2} A\right\|_{n}, \\
& \left\|\mathscr{L}^{-1 / 4} Q_{2}(A)\right\|_{n} \leq M\left\|\mathscr{L}^{1 / 4} A\right\|_{n}^{3} .
\end{aligned}
$$

If $A, B \in D\left(\mathscr{L}^{\alpha}\right)$ for $\frac{1}{2}<\alpha<1$, then

$$
\begin{aligned}
\left\|\mathscr{L}^{-1 / 4}\left(Q_{1}(A)-Q_{1}(B)\right)\right\|_{n} & \leq M\left(\left\|\mathscr{L}^{1 / 4}(A-B)\right\|_{n}\left\|\mathscr{L}^{1 / 2} A\right\|_{n}\right. \\
& \left.+\left\|\mathscr{L}^{1 / 4} A\right\|_{n}\left\|\mathscr{L}^{1 / 2}(A-B)\right\|_{n}\right) \\
\left\|\mathscr{L}^{-1 / 4}\left(Q_{2}(A)-Q_{2}(B)\right)\right\|_{n} & \leq M\left(\left\|\mathscr{L}^{1 / 4} A\right\|_{n}^{2}+\left\|\mathscr{L}^{1 / 4} B\right\|_{n}^{2}\right)\left\|\mathscr{L}^{1 / 4}(A-B)\right\|_{n},
\end{aligned}
$$


where the constant $M$ is independent of $A$ and $B$.

Proof. It is easy to see that $\mathscr{L}_{r}^{*}$, the adjoint operator of $\mathscr{L}_{r}$ in $L^{r}\left(\Omega^{1}\left(\mathfrak{g}_{P}\right)\right)$, satisfies $\mathscr{L}_{r}^{*}=\mathscr{L}_{r^{\prime}}$, where $1 / r+1 / r^{\prime}=1$.

Take $r \in(1, \infty)$ so that $1 / r=1 / n+1 / 2 n$. Then by (3.4) we have $\|A\|_{r^{\prime}}$ $\leq C\left\|\mathscr{L}_{n^{\prime}}^{1 / 4} A\right\|_{n^{\prime}}$ for all $A \in D\left(\mathscr{L}_{n^{\prime}}^{1 / 4}\right)$ with $C$ independent of $A\left(n^{\prime}=\frac{n}{n-1}\right)$. Hence Hölder's inequality yields

$$
\begin{aligned}
\left|\left\langle\mathscr{L}^{-1 / 4} Q_{1}(A), \varphi\right\rangle\right| & =\left|\left\langle Q_{1}(A), \mathscr{L}_{n^{\prime}}^{-1 / 4} \varphi\right\rangle\right| \\
& \leq\left\|Q_{1}(A)\right\|_{r}\left\|\mathscr{L}_{n^{\prime}}^{-1 / 4} \varphi\right\|_{r^{\prime}} \\
& \leq C\|A\|_{2 n}\|\nabla A\|_{n}\left\|\mathscr{L}_{n}^{1 / 4} \mathscr{L}_{n^{\prime}}^{-1 / 4} \varphi\right\|_{n^{\prime}} \\
& \leq M\left\|\mathscr{L}^{1 / 4} A\right\|_{n}\left\|\mathscr{L}^{1 / 2} A\right\|_{n}\|\varphi\|_{n^{\prime}}
\end{aligned}
$$

for all $\varphi \in \Omega^{1}\left(\mathfrak{g}_{P}\right)$. By duality, we obtain

$$
\left\|\mathscr{L}^{-1 / 4} Q_{1}(A)\right\|_{n} \leq M\left\|\mathscr{L}^{1 / 4} A\right\|_{n}\left\|\mathscr{L}^{1 / 2} A\right\|_{n} .
$$

Similarly, we have for $r=\frac{2 n}{3}$.

$$
\begin{aligned}
\left|\left\langle\mathscr{L}^{-1 / 4} Q_{2}(A), \varphi\right\rangle\right| & \leq\left\|Q_{2}(A)\right\|_{r}\|\varphi\|_{n^{\prime}} \\
& \leq C\|A\|_{3 r}^{3}\|\varphi\|_{n^{\prime}}=C\|A\|_{2 n}^{3}\|\varphi\|_{n^{\prime}} \leq M\left\|\mathscr{L}^{1 / 4} A\right\|_{n}^{3}\|\varphi\|_{n^{\prime}},
\end{aligned}
$$

for all $\varphi \in \Omega^{1}\left(\mathfrak{g}_{P}\right)$, from which it follows that

$$
\left\|\mathscr{L}^{-1 / 4} Q_{2}(A)\right\|_{n} \leq M\left\|\mathscr{L}^{1 / 4} A\right\|_{n}^{3} .
$$

Using (3.7), we easily get (3.8).

Lemma 3.4. Let $A^{(0)} \in L^{n}\left(\Omega^{1}\left(\mathfrak{g}_{P}\right)\right)$. Then there exist $T>0$ and a function $A(t)$ on $[0, T)$ such that $A \in C\left([0, T) ; L^{n}\left(\Omega^{1}\left(\mathfrak{g}_{p}\right)\right)\right) \cap C\left((0, T) ; D\left(\mathscr{L}^{\alpha}\right)\right)$ with

$$
\sup _{0<t<T} t^{\alpha}\left\|\mathscr{L}^{\alpha} A(t)\right\|_{n}<\infty \text { for } 0 \leq \alpha<\frac{3}{4}
$$

$A$ is a solution of the integral equation

(3.10) $A(t)=e^{-t \mathscr{L}} A^{(0)}-\int_{0}^{t} e^{-(t-s) \mathscr{L}} d_{D_{0}}^{*} R d s-\int_{0}^{t} e^{-(t-s) \mathscr{L}} Q(A)(s) d s, 0 \leq t \leq T$.

Proof. We solve (3.10) by the following successive approximation: 


$$
\left\{\begin{array}{c}
A_{1}(t)=e^{-t \mathscr{L}} A^{(0)}-\int_{0}^{t} e^{-(t-s) \mathscr{L}} d_{D_{0}}^{*} R d s, \\
A_{j+1}(t)=A_{1}(t)-\int_{0}^{t} e^{-(t-s) \mathscr{L}} Q\left(A_{j}\right)(s) d s, j=1,2, \cdots
\end{array}\right.
$$

Let us first show that

$$
\sup _{0<t<T} t^{\alpha}\left\|\mathscr{L}^{\alpha} A_{j}(t)\right\|_{n} \leq K_{\alpha, j}, \quad 0 \leq \alpha<\frac{3}{4}, j=1,2, \cdots,
$$

Indeed for $j=1$, we have

$$
\begin{aligned}
t^{\alpha}\left\|\mathscr{L}^{\alpha} A_{1}(t)\right\|_{n} & \leq t^{\alpha}\left\|\mathscr{L}^{\alpha} e^{-t \mathscr{L}} A^{(0)}\right\|_{n}+t^{\alpha} \int_{0}^{t}\left\|e^{-(t-s) \mathscr{L}} d_{D_{0}}^{*} R\right\|_{n} d s \\
& \leq\left\|A^{(0)}\right\|_{n}+t^{\alpha} \lambda_{*}^{-1}\left\|d_{D_{0}}^{*} R\right\|_{n} \\
& \leq\left\|A^{(0)}\right\|_{n}+\lambda_{*}^{-1}\left\|d_{D_{0}}^{*} R\right\|_{n},
\end{aligned}
$$

where $\lambda_{*}$ is the smallest eigenvalue of $\mathscr{L}$ and hence we may take

$$
K_{\alpha, 1}:=\sup _{0<t<T} t^{\alpha}\left\|\mathscr{L}^{\alpha} e^{-t \mathscr{L}} A^{(0)}\right\|_{n}+T^{\alpha} \lambda_{*}^{-1}\left\|d_{D_{0}}^{*} R\right\|_{n},
$$

for all $0 \leq \alpha<\frac{3}{4}$.

Suppose that (3.12) holds for $j$. Then from Lemma 3.3, we have

$$
\begin{aligned}
\left\|\mathscr{L}^{\alpha} A_{j+1}(t)\right\|_{n} & \leq\left\|\mathscr{L}^{\alpha} A_{1}(t)\right\|_{n}+\int_{0}^{t}\left\|\mathscr{L}^{\alpha+1 / 4} e^{-(t-s) \mathscr{L}} \mathscr{L}^{-1 / 4} Q\left(A_{j}\right)(s)\right\|_{n} d s \\
& \leq K_{\alpha, 1} t^{-\alpha}+\int_{0}^{t}(t-s)^{-\alpha-1 / 4}\left\|\mathscr{L}^{-1 / 4} Q\left(A_{j}\right)(s)\right\|_{n} d s \\
& \leq K_{\alpha, 1} t^{-\alpha} \\
& +M \int_{0}^{t}(t-s)^{-\alpha-1 / 4}\left(\left\|\mathscr{L}^{1 / 4} A_{j}(s)\right\|_{n}\left\|\mathscr{L}^{1 / 2} A_{j}(s)\right\|_{n}+\left\|\mathscr{L}^{1 / 4} A_{j}(s)\right\|_{n}^{3}\right) d s \\
& \leq K_{\alpha, 1} t^{-\alpha}+M\left(K_{1 / 4, j} K_{1 / 2, j}+K_{1 / 4, j}^{3}\right) \int_{0}^{t}(t-s)^{-\alpha-1 / 4} s^{-3 / 4} d s \\
& \leq K_{\alpha, 1} t^{-\alpha}+M B(3 / 4-\alpha, 1 / 4)\left(K_{1 / 4, j} K_{1 / 2, j}+K_{1 / 4, j}^{3}\right) t^{-\alpha}
\end{aligned}
$$

for $0 \leq \alpha<3 / 4$ and $0<t<T$, where $B(\cdot, \cdot)$ denotes the beta function. Hence (3.12) is satisfied with $j$ replaced by $j+1$, with

$$
K_{\alpha, \jmath+1}:=K_{\alpha, 1}+M B(3 / 4-\alpha, 1 / 4)\left(K_{1 / 4, j} K_{1 / 2, j}+K_{1 / 4, j}^{3}\right) .
$$

(3.13) shows that $\left\{K_{\alpha, j}\right\}_{j=1}^{\infty}$ is a closed recurrence for $\alpha=\frac{1}{4}$ and $\alpha=\frac{1}{2}$. Now let 
$k_{j}:=\max \left\{K_{1 / 4, j}, K_{1 / 2, j}\right\}(j=1,2, \cdots)$. Then by (3.13), we have

$$
k_{j+1} \leq k_{1}+2 M \beta\left(k_{j}^{2}+k_{j}^{3}\right), \beta=B(1 / 4,1 / 4) .
$$

for $j=1,2, \cdots$. By (3.14), we see that there exist positive constants $m_{*}$ and $k$ such that if

$$
k_{1}<m_{*}
$$

then

$$
k_{j} \leq k \text { for all } j=1,2, \cdots .
$$

In fact, $m_{*}$ is determined by the local maximum of the function $f(x)=x-$ $2 M \beta\left(x^{2}+x^{3}\right)$ and $k$ is the positive root of the equation $f(x)=k_{1}$.

Assume (3.15) for a moment and set

$$
B_{j}(t):=A,(t)-A_{j-1}(t), j=1,2, \cdots,\left(A_{0}(t)=0\right) .
$$

From (3.8) and (3.16), we have

$$
\begin{aligned}
\left\|\mathscr{L}^{\alpha} B_{j+1}(t)\right\|_{n} & \leq \int_{0}^{t}\left\|\mathscr{L}^{\alpha+1 / 4} e^{-(t-s) \mathscr{L}} \mathscr{L}^{-1 / 4}\left(Q\left(A_{j}\right)(s)-Q\left(A_{j-1}\right)(s)\right)\right\|_{n} d s \\
& \leq \int_{0}^{t}\left\|\mathscr{L}^{\alpha+1 / 4} e^{-(t-s) \mathscr{L}}\right\|_{B\left(L^{n}\right)}\left\|\mathscr{L}^{-1 / 4}\left(Q\left(A_{j}\right)(s)-Q\left(A_{j-1}\right)(s)\right)\right\|_{n} d s \\
& \leq M \int_{0}^{t}(t-s)^{-\alpha-1 / 4}\left\{\left\|\mathscr{L}^{1 / 2} B_{j}(s)\right\|_{n}\left\|\mathscr{L}^{1 / 4} A_{j}(s)\right\|_{n}\right. \\
& +\left\|\mathscr{L}^{1 / 2} A_{j-1}(s)\right\|_{n}\left\|\mathscr{L}^{1 / 4} B_{j}(s)\right\|_{n} \\
& \left.+\left(\left\|\mathscr{L}^{1 / 4} A_{j}(s)\right\|_{n}^{2}+\left\|\mathscr{L}^{1 / 4} A_{j-1}\right\|_{n}^{2}\right)\left\|\mathscr{L}^{1 / 4} B_{j}(s)\right\|_{n}\right\} d s \\
& \leq M k \int_{0}^{t}(t-s)^{-\alpha-1 / 4}\left(\left\|\mathscr{L}^{1 / 2} B_{j}(s)\right\|_{n} s^{-1 / 4}+\left\|\mathscr{L}^{1 / 4} B_{j}(s)\right\|_{n} s^{-1 / 2}\right) d s \\
& +2 M k^{2} \int_{0}^{t}(t-s)^{-\alpha-1 / 4}\left\|\mathscr{L}^{1 / 2} B_{j}(s)\right\|_{n} s^{-1 / 4} d s
\end{aligned}
$$

for $0 \leq \alpha<\frac{3}{4}$.

Taking $\alpha=1 / 4$ and $\alpha=1 / 2$ in (3.17), we get by induction

$$
\left\{\begin{array}{l}
\left\|\mathscr{L}^{1 / 4} B_{j}(t)\right\|_{n} \leq k\left\{2 M \beta\left(k+k^{2}\right)\right\}^{j-1} t^{-1 / 4}, \\
\left\|\mathscr{L}^{1 / 2} B_{j}(t)\right\|_{n} \leq k\left\{2 M \beta\left(k+k^{2}\right)\right\}^{j-1} t^{-1 / 2}, j=1,2, \cdots
\end{array}\right.
$$

By (3.17) and (3.18), 


$$
\left\|\mathscr{L}^{\alpha} B_{j+1}(t)\right\|_{n} \leq k\left\{2 M \beta\left(k+k^{2}\right)\right\}^{j-1}\left\{2 M B\left(\frac{3}{4}-\alpha, \frac{1}{4}\right)\left(k+k^{2}\right)\right\} t^{-\alpha},
$$

$(0<t<T)$.

Since $k$ satisfies $k_{1}=k-2 M \beta\left(k^{2}+k^{3}\right)$, under the assumption (3.15) we have $2 M \beta\left(k+k^{2}\right)=1-k_{1} / k \in(0,1)$ and hence by (3.19) the sequence $A_{j}(t)=\sum_{r=1}^{j} B_{r}(t)$ converges absolutely and uniformly in $L^{n}\left(\Omega^{1}\left(\mathrm{~g}_{P}\right)\right)$ with respect to $[0, T): A_{j}(t) \rightarrow A(t)$, where $A \in B C\left([0, T) ; L^{n}\left(\Omega^{1}\left(\mathfrak{g}_{p}\right)\right)\right)$.

Moreover, again by (3.19), for each $0<\alpha<\frac{3}{4}$ there exists $A^{(\alpha)} \in C((0, T)$; $\left.L^{n}\left(\Omega^{1}\left(\mathfrak{g}_{P}\right)\right)\right)$ with $t^{\alpha} A^{(\alpha)}(t) \in B C\left([0, T) ; L^{n}\left(\Omega^{1}\left(\mathfrak{g}_{P}\right)\right)\right)$ such that

$$
\sup _{0<t<T} t^{\alpha}\left\|\mathscr{L}^{\alpha} A_{j}(t)-A^{(\alpha)}(t)\right\|_{n} \rightarrow 0 \quad \text { as } j \rightarrow \infty .
$$

Since $\mathscr{L}$ is a closed operator on $L^{n}\left(\Omega^{1}\left(\mathfrak{g}_{p}\right)\right)$, we can conclude that $A \in C((0, T)$; $\left.D\left(\mathscr{L}^{\alpha}\right)\right)$ with $\mathscr{L}^{\alpha} A(t)=A^{(\alpha)}(t)$ for all $0<t<T$, and hence

$$
\sup _{0<t<T} t^{\alpha}\left\|\mathscr{L}^{\alpha}\left(A_{j}(t)-A(t)\right)\right\|_{n} \rightarrow 0,\left(0 \leq \alpha<\frac{3}{4}\right) \text { as } j \rightarrow \infty .
$$

Now again by (3.8), (3.16) and (3.20),

$$
\begin{aligned}
& \left\|\int_{0}^{t} e^{-(t-s) \mathscr{L}}\left(Q\left(A_{j}\right)(s)-Q(A)(s)\right) d s\right\|_{n} \\
\leq & \int_{0}^{t}\left\|\mathscr{L}^{1 / 4} e^{-(t-s) \mathscr{L}}\right\|_{B\left(L^{n}\right)}\left\|\mathscr{L}^{-1 / 4}\left(Q\left(A_{j}\right)(s)-Q(A)(s)\right)\right\|_{n} d s \\
\leq & M \int_{0}^{t}(t-s)^{-1 / 4}\left\{\left\|\mathscr{L}^{1 / 4}\left(A_{j}(s)-A(s)\right)\right\|_{n}\left\|\mathscr{L}^{1 / 4} A_{j}(s)\right\|_{n}\right. \\
+ & \left\|\mathscr{L}^{1 / 2} A(s)\right\|_{n}\left\|\mathscr{L}^{1 / 4}\left(A_{j}(s)-A(s)\right)\right\|_{n} \\
+ & \left.\left(\left\|\mathscr{L}^{1 / 4} A_{j}(s)\right\|_{n}^{2}+\left\|\mathscr{L}^{1 / 4} A_{j}(s)\right\|_{n}^{2}\right)\left\|\mathscr{L}^{1 / 4}\left(A_{j}(s)-A(s)\right)\right\|_{n}\right\} d s \\
\leq & M k \beta \sup _{0<s<t} s^{1 / 2}\left\|\mathscr{L}^{1 / 2}\left(A_{j}(s)-A(s)\right)\right\|_{n} \\
+ & \left(M k \beta+2 M k^{2} \beta\right) \sup _{0<s<t} s^{1 / 4}\left\|\mathscr{L}^{1 / 4}\left(A_{j}(s)-A(s)\right)\right\|_{n} \\
\rightarrow & 0 \text { as } j \rightarrow \infty
\end{aligned}
$$

Under the assumption (3.15), we see by (3.11) and the above convergence that $A$ is the desired solution of (3.10).

It remains to show that we can take $T$ so small that $(3.15)$ is satisfied. Since $D\left(\mathscr{L}^{1 / 2}\right)$ is dense in $L^{n}\left(\Omega^{1}\left(\mathfrak{g}_{P}\right)\right)$, there exists a function $\tilde{A}^{(0)} \in D\left(\mathscr{L}^{1 / 2}\right)$ such that 
$\left\|A^{(0)}-\tilde{A}^{(0)}\right\|_{n}<\frac{m_{*}}{2}$. Then, we have

$$
\begin{aligned}
t^{\alpha}\left\|\mathscr{L}^{\alpha} e^{-t \mathscr{L}} A^{(0)}\right\|_{n} & \leq t^{\alpha}\left\|\mathscr{L}^{\alpha} e^{-t \mathscr{L}}\left(A^{(0)}-\tilde{A}^{(0)}\right)\right\|_{n}+t^{\alpha}\left\|\mathscr{L}^{\alpha} e^{-t \mathscr{L}} \tilde{A}^{(0)}\right\|_{n} \\
& \leq\left\|A^{(0)}-\tilde{A}^{(0)}\right\|_{n}+t^{\alpha}\left\|\mathscr{L}^{\alpha} \tilde{A}^{(0)}\right\|_{n} \\
& \leq \frac{m_{*}}{2}+t^{\alpha}\left\|\mathscr{L}^{\alpha} \tilde{A}^{(0)}\right\|_{n}, t>0,
\end{aligned}
$$

for $\alpha=1 / 4$ and $\alpha=1 / 2$. Since

$$
K_{\alpha, 1}=\sup _{0<t<T} t^{\alpha}\left\|\mathscr{L}^{\alpha} e^{-t \mathscr{L}} A^{(0)}\right\|_{n}+T^{\alpha} \lambda_{*}^{-1}\left\|d_{D_{0}}^{*} R\right\|_{n},
$$

$T$ may be taken to be

$$
T=\min \left\{\left(\frac{m_{*}}{2\left(\left\|\mathscr{L}^{1 / 2} \tilde{A}^{(0)}\right\|_{n}+\lambda_{*}^{-1}\left\|d_{D_{0}}^{*} R\right\|_{n}\right)}\right)^{1 / \alpha}: \alpha=\frac{1}{4}, \frac{1}{2}\right\} .
$$

This completes the proof of Lemma 3.4.

Let us show that $A$ in Lemma 3.4 also satisfies (1), (2) and (3) in Theorem 3.1 .

Lemma 3.5. Let $A$ be the solution of (3.10) given by Lemma 3.4. Then for $0 \leq \alpha<\frac{3}{4}$, the function $\mathscr{L}^{\alpha} A(t)$ is a Hölder continuous on $(0, T)$ with values in $L^{n}\left(\Omega^{1}\left(\mathrm{~g}_{P}\right)\right)$. More precisely, for $0 \leq \alpha<\frac{3}{4}$ there exists $0<\eta<\frac{3}{4}-\alpha$ such that

$$
\left\|\mathscr{L}^{\alpha} A(t+h)-\mathscr{L}^{\alpha} A(t)\right\|_{n} \leq C\left(h^{n} t^{-\alpha-\eta}+h^{3 / 4-\alpha} t^{-3 / 4}+h^{1-\alpha}\right)
$$

holds for all $h>0$ and $0<t<T-h$, where $C=C(\alpha, \eta, M, k)$ is independent of $h$ and $t$.

Proof. An elementary calculation shows

$$
\left\|\left(e^{-h \mathscr{L}}-1\right) A\right\|_{n} \leq C h^{\gamma}\left\|\mathscr{L}^{\gamma} A\right\|_{n}, \quad A \in D\left(\mathscr{L}^{\gamma}\right), \quad 0<\gamma<1,
$$

for all $h>0$. By Lemma 3.3, (3.12) and (3.16), we get

$$
\begin{aligned}
& \left\|\mathscr{L}^{\alpha} A(t+h)-\mathscr{L}^{\alpha} A(t)\right\|_{n} \leq\left\|\left(e^{-h \mathscr{L}}-1\right) \mathscr{L}^{\alpha} e^{-t \mathscr{L}} A^{(0)}\right\|_{n} \\
& +\int_{t}^{t+h}\left\|\mathscr{L}^{\alpha} e^{-(t+h-s) \mathscr{L}} d_{D_{0}}^{*} R\right\|_{n} d s
\end{aligned}
$$




$$
\begin{aligned}
& +\int_{0}^{t}\left\|\left(e^{-h \mathscr{L}}-1\right) \mathscr{L}^{\alpha} e^{-(t-s) \mathscr{L}} d_{D_{0}}^{*} R\right\|_{n} d s \\
& +\int_{t}^{t+h}\left\|\mathscr{L}^{\alpha+1 / 4} e^{-(t+h-s) \mathscr{L}} \mathscr{L}^{-1 / 4} Q(A)(s)\right\|_{n} d s \\
& +\int_{0}^{t}\left\|\left(e^{-h \mathscr{L}}-1\right) \mathscr{L}^{\alpha+1 / 4} e^{-(t-s) \mathscr{L}} \mathscr{L}^{-1 / 4} Q(A)(s)\right\|_{n} d s \\
& \leq C h^{\eta}\left\|\mathscr{L}^{\alpha+\eta} e^{-h \mathscr{L}} A^{(0)}\right\|_{n}+C\left(h^{1-\alpha}+h^{\eta} t^{1-\alpha+\eta}\right)\left\|d_{D_{0}}^{*} R\right\|_{n} \\
& +\int_{t}^{t+h}(t+h-s)^{-\alpha-1 / 4}\left(\left\|\mathscr{L}^{1 / 2} A(s)\right\|_{n}\left\|\mathscr{L}^{1 / 4} A(s)\right\|_{n}+\left\|\mathscr{L}^{1 / 4} A(s)\right\|_{n}^{3}\right) d s \\
& +C h^{\eta} \int_{0}^{t}(t-s)^{-\alpha-1 / 4-\eta}\left(\left\|\mathscr{L}^{1 / 2} A(s)\right\|_{n}\left\|\mathscr{L}^{1 / 4} A(s)\right\|_{n}+\left\|\mathscr{L}^{1 / 4} A(s)\right\|_{n}^{3}\right) d s \\
& \leq C h^{\eta} t^{-\alpha-\eta}\left\|A^{(0)}\right\|_{n}+C\left(h^{1-\alpha}+h^{\eta} t^{1-\alpha-\eta}\right)\left\|d_{D_{0}}^{*} R\right\|_{n} \\
& +C M\left(k^{2}+k^{3}\right) \int_{t}^{t+h}(t+h-s)^{-\alpha-1 / 4} s^{-3 / 4} d s \\
& +C M\left(k^{2}+k^{3}\right) h^{\eta} \int_{0}^{t}(t-s)^{-\alpha-1 / 4-\eta} s^{-3 / 4} d s \\
& \leq C h^{\eta} t^{-\alpha-\eta}\left\|A^{(0)}\right\|_{n}+C\left(h^{1-\alpha}+h^{\eta} t^{1-\alpha-\eta}\right)\left\|d_{D_{0}}^{*} R\right\|_{n}+\frac{M\left(k^{2}+k^{3}\right)}{3 / 4-\alpha} h^{3 / 4-\alpha} t^{-3 / 4} \\
& +C M\left(k^{2}+k^{3}\right) B(3 / 4-\alpha-\eta, 1 / 4) h^{\eta} t^{-\alpha-\eta}, 0 \leq \alpha<\frac{3}{4},
\end{aligned}
$$

for all $t>0, h>0$, where $0 \leq \eta<\frac{3}{4}-\alpha$, from which (3.21) follows.

Proof of Theorem 3.1. Let $A(t)$ be the solution of (3.10) given in Lemma 3.4. Then by Lemmas 3.2 and 3.5 , we see that the function $Q(A)(t)$ is Hölder continuous on $(0, T)$ with values in $L^{n}\left(\Omega^{1}\left(g_{P}\right)\right)$. By the general theory of holomorphic semigroups (see e.g. Tanabe [10, Theorem 3.3.2]), $A$ is also a solution of (3.2) in the class of (1) and (2) in Theorem 3.1. This completes the proof.

Remark 3.6. By using a standard argument of semilinear parabolic equations, we can prove that the strong solution $A(t)$ given by Theorem 3.1 is actually smooth (i.e., of class $\left.C^{\infty}\right)$ on $M \times(0, T)$. 


\section{Estimates}

Now we return to the case when $M$ is a closed 4 -manifold. In this section, we give various estimates for the curvature tensor, which will be useful for characterizing the singular set $\&$.

In the following sections, a connection $D(t)$ is smooth means that $D(t) \in C^{\infty}$.

Lemma 4.1. Let $D$ be a smooth solution of (1.1). Then the function

$$
E(t)=\frac{1}{2} \int_{M}\left|R_{D}(\cdot, t)\right|^{2} d V
$$

is non-increasing.

Proof. Taking the $L^{2}$-inner product with $R_{D}$ in (2.4), we have

$$
\frac{d}{d t} \frac{1}{2} \int_{M}\left|R_{D}\right|^{2} d V=-\int_{M}\left|d_{D}^{*} R_{D}\right|^{2} d V \leq 0
$$

for any $t \in[0, T]$, which gives Lemma 4.1 .

By Lemma 4.1 , if $D$ is a solution of (1.1), then for any $t \in[0, T]$,

$$
E(t)=\int_{M}\left|R_{D}(\cdot, t)\right|^{2} d V
$$

is bounded from above. For a smooth solution $A$, put

$$
\varepsilon(r)=\varepsilon(r, x)=\sup _{0 \leq t \leq T}\left(\int_{B_{r}(x)}\left|R_{D}(\cdot, t)\right|^{2} d V\right)^{1 / 2} .
$$

In the sequel we give a priori bounds for the norm of $D$ in terms of the initial energy $E\left(D^{(0)}\right)=E_{0}, T$ and $\varepsilon_{1}$. Here $\varepsilon_{1}>0$ is a parameter depending only on $M$ which will be determined in Lemmas $4.2-4.9$. To obtain these, we use the Sobolev embedding in 4 -dimensional case. We will set $\varepsilon_{1}$ to be the smallest of the numbers $\varepsilon_{1}$ appearing in Lemmas $4.2-4.9$.

Lemma 4.2. Let $R_{0}$ be as in Proposition 2.1. There exists a constant $\varepsilon_{1}>0$ such that for any smooth solution $D$ of (1.1) on $(0, T)$ with the initial value $(1.2), D^{(0)} \in$ $\mathfrak{u}^{1,2}$ and any number $r \in\left(0, R_{0}\right]$, if $\varepsilon(r, x)<\varepsilon_{1}$, then we have 


$$
\int_{0}^{T} \int_{B_{r / 2}(x)}\left|\tilde{\nabla}_{D} R_{D}\right|^{2} d V d t \leq C\left(1+r^{-2} T\right) E_{0},
$$

where the constant $C$ depends only on $M$.

Proof. Let $\varphi$ be a non-increasing with respect to $\operatorname{dist}(x, \cdot)$ real-valued function independent of $t$, which satisfies $\varphi=0$ outside $B_{r}(x), \varphi=1$ on $B_{r / 2}(x)$ and $|\nabla \varphi| \leq c / r$. Taking the $L^{2}$-inner product with $R_{D} \varphi^{2}$ in (2.5), we have

$$
\begin{aligned}
& \frac{1}{2} \frac{d}{d t} \int_{B_{r}(x)}\left|R_{D}\right|^{2} \varphi^{2} d V+\int_{B_{r}(x)}\left|\tilde{\nabla}_{D} R_{D}\right|^{2} \varphi^{2} d V \\
& \leq C \int_{B_{r}(x)}\left|R_{D}\right|^{3} \varphi^{2} d V+C \int_{B_{r}(x)}\left|R_{D}\right|\left|\tilde{\nabla}_{D} R_{D}\right||\nabla \varphi| \varphi d V .
\end{aligned}
$$

For the right hand side of (4.3), using Lemma 2.2, we have

$$
\begin{array}{r}
\int_{B_{r}(x)}\left|R_{D}\right|\left|\tilde{\nabla}_{D} R_{D}\right||\nabla \varphi| \varphi d V \leq \varepsilon \int_{B_{r}(x)}\left|\tilde{\nabla}_{D} R_{D}\right|^{2} \varphi^{2} d V+C r^{-2} \int_{B_{r}(x)}\left|R_{D}\right|^{2} d V \\
\int_{B_{r}(x)}\left|R_{D}\right|^{3} \varphi^{2} d V \leq C\left(\int_{B_{r}(x)}\left|R_{D}\right|^{2} d V\right)^{1 / 2} \cdot\left(\int_{B_{r}(x)}|\nabla| R_{D}||^{2} \varphi^{2} d V+r^{-2} \int_{B_{r}(x)}\left|R_{D}\right|^{2} d V\right) \\
\leq C\left(\int_{B_{r}(x)}\left|R_{D}\right|^{2} d V\right)^{1 / 2} \cdot\left(\int_{B_{r}(x)}\left|\tilde{\nabla}_{D} R_{D}\right|^{2} \varphi^{2} d V+r^{-2} \int_{B_{r}(x)}\left|R_{D}\right|^{2} d V\right) .
\end{array}
$$

Since we assume that $\varepsilon(r)<\varepsilon_{1}$, we obtain

$$
\frac{1}{2} \frac{d}{d t} \int_{B_{\gamma}(x)}\left|R_{D}\right|^{2} \varphi^{2} d V+C \int_{B_{\gamma}(x)}\left|\tilde{\nabla}_{D} R_{D}\right|^{2} \varphi^{2} d V \leq C r^{-2} \int_{B_{\gamma}(x)}\left|R_{D}\right|^{2} d V .
$$

Integrating (4.4) over $[0, T]$, we have

$$
\begin{aligned}
& \frac{1}{2} \int_{B_{r}(x)}\left|R_{D}\right|^{2}(T) \varphi^{2} d V+C \int_{0}^{T} \int_{B_{r}(x)}\left|\tilde{\nabla}_{D} R_{D}\right|^{2} \varphi^{2} d V d t \\
& \leq \frac{1}{2} \int_{B_{r}(x)}\left|R_{D}\right|^{2}(0) d V+C r^{-2} \int_{0}^{T} \int_{B_{r}(x)}\left|R_{D}\right|^{2} d V d t .
\end{aligned}
$$

Therefore we have

$$
\int_{0}^{T} \int_{B_{r / 2}(x)}\left|\tilde{\nabla}_{D} R_{D}\right|^{2} d V d t \leq C\left(1+r^{-1} T\right) E_{0}
$$

Lemma 4.3. Let $R_{0}$ be as in Proposition 2.1. There exists a constant $\varepsilon_{1}>0$ such that for any smooth solution $D$ of $(1.1)$ on $(0, T)$ with the initial value $(1.2), D^{(0)} \in$ 
$\mathfrak{u}^{1,2}$, any $\tau>0$ and any number $r \in\left(0, R_{0}\right]$, if $\varepsilon(r, x)<\varepsilon_{1}$, then we have

$$
\sup _{\substack{\tau<t<T \\ x \in B_{r / 2}(x)}}\left|R_{D}\right| \leq C
$$

where the constant $C$ depends on $\tau, T, r, E_{0}$ and $M$.

Proof. By (2.7) with $n=1$, we have

$$
\partial_{t} v \leq \Delta v+C_{0} u v,
$$

where $u=\left|R_{D}\right|, v=\left|\tilde{\nabla}_{D} R_{D}\right|$. Let $\phi$ be a non-negative function. Multiplying the above inequality by $v^{p} \phi^{2}, p \geq 1$ and integrating over $[0, T] \times B_{r}(x)$, by Lemma 2.2 , we have

$$
\begin{aligned}
& \int_{0}^{T} \int_{B_{\gamma}(x)} \partial_{t}\left(v^{p+1} \phi^{2}\right) d V d t+\frac{4 p}{p+1} \int_{0}^{T} \int_{B_{r}(x)}\left|\tilde{\nabla} v^{\frac{p+1}{2}}\right|^{2} \phi^{2} d V d t \\
& \leq 4 \int_{0}^{T} \int_{B_{\gamma}(x)}\left|\nabla v^{\frac{p+1}{2}}\right| v^{\frac{p+1}{2}}|\nabla \phi| \phi d V d t+2 \int_{0}^{T} \int_{B_{r}(x)} v^{p+1}\left|\partial_{t} \phi\right| \phi d V d t \\
& +C_{0} C_{1}(p+1) \sup _{0 \leq t \leq T}\left(\int_{B_{r}(x)} u^{2} d V\right)^{1 / 2} \\
& \cdot\left[\int_{0}^{T} \int_{B_{\gamma}(x)}\left|\nabla v^{\frac{p+1}{2}}\right|^{2} \phi^{2} d V d t+r^{-2} \int_{0}^{T} \int_{B_{\gamma}(x)} v^{p+1} \phi^{2} d V d t\right] .
\end{aligned}
$$

Let us take $\phi$ such that $\phi(t, y)=\phi_{1}(t) \phi_{2}(\operatorname{dist}(x, y))$, where $\phi_{1}$ is non-decreasing, $\psi_{2}$ is non-increasing and

$$
\phi_{1}(t)=\left\{\begin{array}{l}
0 \quad \text { in } 0 \leq t \leq \delta \tau \\
1 \quad \text { in } \tau \leq t \leq T,
\end{array} \quad \phi_{2}(s)= \begin{cases}1 & \text { in } s<\delta r \\
0 & \text { in } s>r,\end{cases}\right.
$$

where $0<\delta<1$.

$$
\begin{aligned}
& \text { If } \sup _{0 \leq t \leq T}\left(\int_{B_{B}(x)} u^{2} d V\right)^{1 / 2} \leq \frac{p}{(p+1)^{2} C_{0} C_{1}} \text {, then we have } \\
& \int_{0}^{T} \int_{B_{r}(x)} \partial_{t}\left(v^{p+1} \phi^{2}\right) d V d t+\frac{p}{p+1} \int_{0}^{T} \int_{B_{r}(x)}\left|\nabla v^{p+1}\right|^{2} \phi^{2} d V d t
\end{aligned}
$$

$$
\begin{aligned}
& \leq \frac{2(p+1)}{p} \int_{0}^{T} \int_{B_{r}(x)} v^{p+1}|\nabla \phi|^{2} d V d t+2 \int_{0}^{T} \int_{B_{r}(x)} v^{p+1}\left|\partial_{t} \phi\right| \phi d V d t \\
& +C_{0} C_{1}(p+1) E_{0}^{1 / 2} r^{-2} \int_{0}^{T} \int_{B_{r}(x)} v^{p+1} \phi^{2} d V d t
\end{aligned}
$$




$$
\leq C_{p, \delta}\left(r^{-2}+\tau^{-1}\right) \int_{0}^{T} \int_{B_{\gamma}(x)} v^{p+1} d V d t
$$

Then there exists $\sigma \in[\tau, T)$, such that

$$
\begin{aligned}
\sup _{\tau \leq t \leq T} \int_{B_{\gamma / 2}(x)} v^{p+1} d V & \leq\left. 2 \int_{B_{\gamma / 2}(x)} v^{p+1} d V\right|_{t=\sigma} \leq 2 \int_{0}^{\sigma} \int_{B_{\gamma / 2}(x)} \partial_{t}\left(v^{p+1} \phi^{2}\right) d V d t \\
& \leq C_{p, \delta}\left(r^{-2}+\tau^{-1}\right) \int_{0}^{T} \int_{B_{\gamma}(x)} v^{p+1} d V d t .
\end{aligned}
$$

Since the first integral on the left hand side in (4.6) is non-negative, we have

$$
\int_{0}^{T} \int_{B_{r}(x)}\left|\nabla v^{\frac{p+1}{2}}\right|^{2} \phi^{2} d V d t \leq C_{p, \delta}\left(r^{-2}+\tau^{-1}\right) \int_{0}^{T} \int_{B_{r}(x)} v^{p+1} d V d t
$$

Thus, we get

$$
\int_{\tau}^{T} \int_{B_{\gamma / 2}(x)}\left|\nabla v^{\frac{p+1}{2}}\right|^{2} d V d t \leq C_{p, \delta}\left(r^{-2}+\tau^{-1}\right) \int_{0}^{T} \int_{B_{\gamma}(x)} v^{p+1} d V d t .
$$

Using Proposition 2.1 and applying (4.7) and (4.8) with $p=1$ with $\delta=1 / \sqrt{2}$ and $p=2$ with $\delta=1 / 2$, we have

$$
\int_{\tau}^{T} \int_{B_{\gamma}(x)} v^{9 / 2} d V d t \leq C\left(r^{-2}+\tau^{-1}\right)^{15 / 4}\left(\int_{0}^{T} \int_{B_{r}(x)} v^{2} d V d t\right)^{9 / 4} .
$$

Combining (4.9) and Lemma 4.2, if $\varepsilon(r, x)<\varepsilon_{1}$, then we have

$$
\int_{\tau}^{T} \int_{B_{r / 2}(x)}\left|\tilde{\nabla}_{D} R_{D}\right|^{9 / 2} d V d t \leq C\left(r^{-2}+\tau^{-1}\right)^{15 / 4}\left(\left(1+r^{-2} T\right) E_{0}\right)^{9 / 4}
$$

Since $9 / 2>\operatorname{dim} M=4$, by Sobolev's embedding and (4.7) with $p=7 / 2$, we obtain

$$
\begin{aligned}
\sup _{\substack{\tau \leq t \leq T \\
B_{r / 2}(x)}}\left|R_{D}\right| & \leq C\left|B_{r / 2}(x)\right|^{1 / 4-2 / 9} \sup _{\tau \leq t \leq T}\left(\int_{B_{r / 2}(x)}\left|\tilde{\nabla}_{D} R_{D}\right|^{9 / 2} d V\right)^{2 / 9} \\
& \leq C\left|B_{r / 2}(x)\right|^{1 / 4-2 / 9}\left(\left(r^{-2}+\tau^{-1}\right) \int_{\tau / 2}^{T} \int_{B_{r}(x)}\left|\tilde{\nabla}_{D} R_{D}\right|^{9 / 2} d V d t\right)^{2 / 9} \\
& \leq C r^{1 / 9}\left(r^{-2}+\tau^{-1}\right)^{19 / 18}\left(\left(1+r^{-2} T\right) E_{0}\right)^{1 / 2}
\end{aligned}
$$

Thus, we get the desired estimate.

Lemma 4.4. Let $R_{0}$ be as in Proposition 2.1. There exists a constant $\varepsilon_{1}>0$ such 
that for any smooth solution $D$ of $(1.1)$ on $(0, T)$ with the initial value $(1.2), D^{(0)} \in$ $\mathfrak{u}^{1,2}$, any $\tau>0$ and any number $r \in\left(0, R_{0}\right]$, if $\varepsilon(r, x)<\varepsilon_{1}$, then we have

$$
\sup _{\substack{\tau<t<T \\ x \in B_{r / 2}(x)}}\left|\tilde{\nabla}_{D} R_{D}\right| \leq C,
$$

where the constant $C$ depends on $\tau, T, r, E_{0}$ and $M$.

Proof. By Lemma 4.3 and (2.7) with $n=1$, for any $\rho$, with $0<\rho<\tau$, we have

$$
\partial_{t}\left|\tilde{\nabla}_{D} R_{D}\right| \leq \Delta\left|\tilde{\nabla}_{D} R_{D}\right|+C\left|\tilde{\nabla}_{D} R_{D}\right|, \quad \text { on }[\rho, T) \times B_{r / 2}(x) .
$$

Note that the constant $C$ depends on $\tau, T, r, E_{0}$ and $M$. Therefore by a Moser's result [5, Theorem 3], we obtain Lemma 4.4 .

Lemma 4.5. Let $R_{0}$ be as in Proposition 2.1. There exists a constant $\varepsilon_{1}>0$ such that for any smooth solution $D$ of (1.1) on $(0, T)$ with the initial value (1.2), $D^{0} \in$ $\mathfrak{u}^{1,2}$, any $n \geq 2$ any $\tau>0$ and any number $r \in\left(0, R_{0}\right]$, if $\varepsilon(r, x)<\varepsilon_{1}$, then we have

$$
\int_{\tau}^{T} \int_{B_{\gamma / 2}(x)}\left|\tilde{\nabla}_{D}^{(n)} R_{D}\right|^{2} d V d t \leq C
$$

where the constant $C$ depends on $n, \tau, T, r, E_{0}, M$ and $\left\|R_{D}(\tau)\right\|_{W^{n, 2}\left(B_{r}(x)\right)}$.

Proof. Let $\varphi$ be the function defined in the proof of Lemma 4.2. Multiplying (2.8) by $\tilde{\nabla}_{D}^{(n)} R_{D} \varphi^{2}$ and integrating over $B_{r}(x)$, we have

$$
\begin{aligned}
& \frac{1}{2} \frac{d}{d t} \int_{B_{r}(x)}\left|\tilde{\nabla}_{D}^{(n)} R_{D}\right|^{2} \varphi^{2} d V+\int_{B_{r}(x)}\left|\tilde{\nabla}_{D}^{(n+1)} R_{D}\right|^{2} \varphi^{2} d V \\
& \leq C \int_{B_{\gamma}(x)}\left|\tilde{\nabla}_{D}^{(n)} R_{D}\right|\left|\tilde{\nabla}_{D}^{(n+1)} R_{D}\right||\nabla \varphi| \varphi d V \\
& +C \sum_{i=0}^{n} \int_{B_{r}(x)}\left|\tilde{\nabla}_{D}^{(i)} R_{D}\right|\left|\tilde{\nabla}_{D}^{(n-i)} R_{D}\right|\left|\tilde{\nabla}_{D}^{(n)} R_{D}\right| \varphi^{2} d V \\
& \leq \varepsilon \int_{B_{r}(x)}\left|\tilde{\nabla}_{D}^{(n+1)} R_{D}\right|^{2} \varphi^{2} d V+C_{\varepsilon} \int_{B_{r}(x)}\left|\tilde{\nabla}_{D}^{(n)} R_{D}\right|^{2}|\nabla \varphi|^{2} d V \\
& +C \sum_{i=0}^{n} \int_{B_{r}(x)}\left|\tilde{\nabla}_{D}^{(i)} R_{D}\right|\left|\tilde{\nabla}_{D}^{(n-i)} R_{D}\right|\left|\tilde{\nabla}_{D}^{(n)} R_{D}\right| \varphi^{2} d V
\end{aligned}
$$


on $[\tau, T)$, for any $\varepsilon>0$.

On the other hand, by Sobolev's embedding, we have

$$
\begin{aligned}
& \sum_{i=0}^{n} \int_{B_{r}(x)}\left|\tilde{\nabla}_{D}^{(i)} R_{D}\right|\left|\tilde{\nabla}_{D}^{(n-i)} R_{D}\right|\left|\tilde{\nabla}_{D}^{(n)} R_{D}\right| \varphi^{2} d V \\
& \leq \sup _{(\tau, T) \times B_{r}(x)}\left|R_{D}\right| \int_{B_{r}(x)}\left|\tilde{\nabla}_{D}^{(n)} R_{D}\right|^{2} \varphi^{2} d V+C \int_{B_{r}(x)}\left|\tilde{\nabla}_{D}^{(n)} R_{D}\right|^{2} \varphi^{2} d V \\
& +C \sum_{i=1}^{n-1} \int_{B_{r}(x)}\left|\tilde{\nabla}_{D}^{(i)} R_{D}\right|^{2}\left|\tilde{\nabla}_{D}^{(n-i)} R_{D}\right|^{2} \varphi^{2} d V \\
& \leq \sup _{(\tau, T) \times B_{r}(x)}\left|R_{D}\right| \int_{B_{r}(x)}\left|\tilde{\nabla}_{D}^{(n)} R_{D}\right|^{2} \varphi^{2} d V+C \int_{B_{r}(x)}\left|\tilde{\nabla}_{D}^{(n)} R_{D}\right|^{2} \varphi^{2} d V \\
& +C \sum_{i=1}^{n-1}\left(\int_{B_{r}(x)}\left|\tilde{\nabla}_{D}^{(i+1)} R_{D}\right|^{2} \varphi^{2} d V+\int_{B_{r}(x)}\left|\tilde{\nabla}_{D}^{(i)} R_{D}\right|^{2}|\nabla \varphi|^{2} d V\right) .
\end{aligned}
$$

Combining Lemma 4.3 with (4.11) and (4.12), we have

$$
\begin{aligned}
& \frac{1}{2} \frac{d}{d t} \int_{B_{\gamma}(x)}\left|\tilde{\nabla}_{D}^{(n)} R_{D}\right|^{2} \varphi^{2} d V+(1-\varepsilon) \int_{B_{r}(x)}\left|\tilde{\nabla}_{D}^{(n+1)} R_{D}\right|^{2} \varphi^{2} d V \\
& \leq C \int_{B_{r}(x)}\left|\tilde{\nabla}_{D}^{(n)} R_{D}\right|^{2} \varphi^{2} d V+C \int_{B_{r}(x)}\left|\tilde{\nabla}_{D}^{(n)} R_{D}\right|^{2}|\nabla \varphi|^{2} d V \\
& +C \sum_{i=1}^{n-1}\left(\int_{B_{r}(x)}\left|\tilde{\nabla}_{D}^{(i+1)} R_{D}\right|^{2} \varphi^{2} d V+\int_{B_{r}(x)}\left|\tilde{\nabla}_{D}^{(i)} R_{D}\right|^{2}|\nabla \varphi|^{2} d V\right) .
\end{aligned}
$$

Integrate (4.13) over $(\tau, T)$, we have

$$
\begin{aligned}
& \frac{1}{2} \int_{B_{\gamma}(x)}\left|\tilde{\nabla}_{D}^{(n)} R_{D}\right|^{2}(T) d V+(1-\varepsilon) \int_{\tau}^{T} \int_{B_{\gamma / 2}(x)}\left|\tilde{\nabla}_{D}^{(n+1)} R_{D}\right|^{2} d V d t \\
& \leq C\left(1+r^{-2}\right) \int_{\tau}^{T} \int_{B_{\gamma}(x)}\left|\tilde{\nabla}_{D}^{(n)} R_{D}\right|^{2} d V d t \\
& +C \sum_{i=1}^{n-1}\left(\int_{\tau}^{T} \int_{B_{\gamma}(x)}\left|\tilde{\nabla}_{D}^{(i+1)} R_{D}\right|^{2} d V d t+r^{-2} \int_{\tau}^{T} \int_{B_{\gamma}(x)}\left|\tilde{\nabla}_{D}^{(i)} R_{D}\right|^{2} d V d t\right) \\
& +\int_{B_{r}(x)}\left|\tilde{\nabla}_{D}^{(n)} R_{D}\right|^{2}(\tau) d V .
\end{aligned}
$$

Since we assume that $R_{D}$ is smooth on $(\tau, T$ ], that last term of (4.14) is bounded. Assume that the inequality in Lemma 4.5 holds for $n$, then by the induction, the right hand side of (4.14) is bounded. Thus, we get Lemma 4.5 in general. 
Lemma 4.6. Let $R_{0}$ be as in Proposition 2.1. There exists a constant $\varepsilon_{1}>0$ such that for any smooth solution $D$ of $(1.1)$ on $(0, T)$ with the initial value $(1.2), D^{(0)} \in$ $\mathfrak{u}^{1,2}$, any $n \geq 2$ any $\tau>0$ and any number $r \in\left(0, R_{0}\right]$, if $\varepsilon(r, x)<\varepsilon_{1}$, then we have

$$
\sup _{\substack{\tau<t<T \\ x \in B_{r / 2}(x)}}\left|\tilde{\nabla}_{D}^{(n)} R_{D}\right| \leq C,
$$

where the constant $C$ depends on $n, \tau, T, r, E_{0}, M$ and $\left\|R_{D}(\tau)\right\|_{W^{n, 2}\left(B_{r}(x)\right)}$.

Proof. By (2.7), we have

$$
\partial_{t}\left|\tilde{\nabla}_{D}^{(n)} R_{D}\right| \leq \Delta\left|\tilde{\nabla}_{D}^{(n)} R_{D}\right|+C\left|R_{D}\right|\left|\tilde{\nabla}_{D}^{(n)} R_{D}\right|+C \sum_{i=1}^{n-1}\left|\tilde{\nabla}_{D}^{(n-\imath)} R_{D}\right|\left|\tilde{\nabla}_{D}^{(\imath)} R_{D}\right|
$$

Using Lemma 4.3 and Lemma 4.5, we may rewrite the inequality (4.15) as

$$
\partial_{t} u \leq \Delta u+C u+C f, \quad \text { on }(\rho, T) \times B_{r / 2}(x),
$$

where $u=\left|\tilde{\nabla}_{D}^{(n)} R_{D}\right| \in L^{2}\left((\rho, T) \times B_{r / 2}(x)\right)$ and $f=\sum_{i=1}^{n-1}\left|\tilde{\nabla}_{D}^{(n-i)} R_{D}\right|\left|\tilde{\nabla}_{D}^{(i)} R_{D}\right|$, for any $\rho$ satisfying $0<\rho<\tau$.

Assume that the conclusion of Lemma 4.6 is true for $n-1$, then we have $f \in L^{p}\left((\rho, T) \times B_{r / 2}(x)\right)$, for $p>2$. Applying [4, Theorem III.8.1, p. 192] to (4.16), we get the desired result.

Lemma 4.7. Let $R_{0}$ be as in Proposition 2.1. There exists a constant $\varepsilon_{1}>0$ such that for any smooth solution $D$ of (1.1) on $(0, T)$ with the initial value $(1.2), D^{(0)} \in$ $\mathfrak{u}^{1,2}$, any $\tau>0$, any $p \geq 2$ and any number $r \in\left(0, R_{0}\right]$, if $\varepsilon(r, x)<\varepsilon_{1}$, then we have

$$
\sup _{\tau<t<T} \int_{B_{r / 2}(x)}|A|^{p} d V \leq C,
$$

where $D=d+A$ on $B_{r}(x)$ and the constant $C$ depends on $\tau, T, r, E_{0}, M$, $\left\|R_{D}(\tau)\right\|_{W^{n, 2}\left(B_{r}(x)\right)}$ and $\|A(\tau)\|_{L^{p}\left(B_{r / 2}(x)\right)}$.

Proof. Multiplying (1.1) by $A|A|^{p-2}$ and integrating over $B_{r / 2}(x)$, we have, by Lemma 4.3 ,

$$
\begin{aligned}
\frac{d}{d t} \int_{B_{\gamma / 2}(x)}|A|^{p} d V & \leq C \int_{B_{\gamma / 2}(x)}\left|\tilde{\nabla}_{D} R_{D}\right||A|^{p-1} d V \\
& \leq\left(\int_{B_{r / 2}(x)}\left|\tilde{\nabla}_{D} R_{D}\right|^{p} d V\right)^{1 / p}\left(\int_{B_{r / 2}(x)}|A|^{p} d V\right)^{(p-1) / p}
\end{aligned}
$$




$$
\leq \int_{B_{r / 2}(x)}|A|^{p} d V+C, \quad \text { on }(\rho, T)
$$

for any $\rho$ satisfying $0<\rho<\tau$. For the function $U(t)=\int_{B_{r / 2}(x)}|A|^{p} d V$, we have

$$
\frac{d}{d t} U(t) \leq C U(t)+C
$$

Lemma 4.7 follows from Gronwall's inequality.

Lemma 4.8. Let $R_{0}$ be as in Proposition 2.1. There exists a constant $\varepsilon_{1}>0$ such that for any smooth solution $D$ of (1.1) on $(0, T)$ with the initial value $(1.2), D^{(0)} \in$ $\mathfrak{u}^{1,2}$, any $n \geq 1$, any $\tau>0$, any $p \geq 2$ and any number $r \in\left(0, R_{0}\right]$, if $\varepsilon(r, x)<\varepsilon_{1}$, then we have

$$
\sup _{\tau<t<T} \int_{B_{r / 2}(x)}\left|\nabla^{(n)} A\right|^{p} d V \leq C,
$$

where $D=d+A$ on $B_{r}(x)$ and the constant $C$ depends on $n, \tau, T, r, E_{0}, M$, $\left\|R_{D}(\tau)\right\|_{W^{n+1,2}\left(B_{r}(x)\right)}$ and $\|A(\tau)\|_{L^{p}\left(B_{\gamma / 2}(x)\right)}$.

Proof. By a direct computation, we get

$$
\tilde{\nabla}_{D}^{(n)} \partial_{t} A=\partial_{t} \tilde{\nabla}_{D}^{(n)} A+C \sum_{k=1}^{n-1} \sum_{i_{1}+\cdots+i_{k}+j+k=n} \tilde{\nabla}_{D}^{\left(i_{1}\right)} A * \cdots * \tilde{\nabla}_{D}^{\left(i_{k}\right)} A * \tilde{\nabla}_{D}^{(j)} \partial_{t} A .
$$

Using (1.1) and (4.18), we have

$$
\partial_{t} \tilde{\nabla}_{D}^{(n)} A=-\tilde{\nabla}_{D}^{(n)} d_{D}^{*} R_{D}+C \sum^{\prime} \tilde{\nabla}_{D}^{\left(i_{1}\right)} A * \cdots * \tilde{\nabla}_{D}^{\left(i_{k}\right)} A * \tilde{\nabla}_{D}^{(j)} \partial_{t} A
$$

where $\sum^{\prime}=\sum_{k=1}^{n-1} \sum_{i_{1}+\cdots+i_{k}+j+k=n}$. Multiplying (4.19) by $\tilde{\nabla}_{D}^{(n)} A\left|\tilde{\nabla}_{D}^{(n)} A\right|^{p-2}$ and integrating over $B_{r / 2}(x)$, we have, by Lemma 4.3 ,

$$
\begin{aligned}
& \frac{d}{d t} \int_{B_{r / 2}(x)}\left|\tilde{\nabla}_{D}^{(n)} A\right|^{p} d V \leq C \int_{B_{r / 2}(x)}\left|\tilde{\nabla}_{D}^{(n+1)} R_{D}\right|\left|\tilde{\nabla}_{D}^{(n)} A\right|^{p-1} d V \\
& \quad+C \sum^{\prime} \int_{B_{r / 2}(x)}\left|\tilde{\nabla}_{D}^{\left(i_{1}\right)} A\right| \cdots\left|\tilde{\nabla}_{D}^{\left(i_{k}\right)} A\right|\left|\tilde{\nabla}_{D}^{(j)} \partial_{t} A\right|\left|\tilde{\nabla}_{D}^{(n)} A\right|^{p-1} d V
\end{aligned}
$$

(4.20) and Young inequality yield

$$
\frac{d}{d t} \int_{B_{\gamma / 2}(x)}\left|\tilde{\nabla}_{D}^{(n)} A\right|^{p} d V \leq C \int_{B_{r / 2}(x)}\left|\tilde{\nabla}_{D}^{(n)} A\right|^{p} d V+C \int_{B_{r / 2}(x)}\left|\tilde{\nabla}_{D}^{(n+1)} R_{D}\right|^{p} d V
$$




$$
+C \sum^{\prime} \int_{B_{\gamma / 2}(x)}\left|\tilde{\nabla}_{D}^{\left(i_{1}\right)} A\right|^{p} \cdots\left|\tilde{\nabla}_{D}^{\left(i_{k}\right)} A\right|^{p}\left|\tilde{\nabla}_{D}^{(j+1)} R_{D}\right|^{p} d V
$$

Assume that the conclusion of Lemma 4.8 is true for $n-1$. By Lemma 4.6 , the last term of the right hand side of $(4.21)$ is bounded by above on $(\rho, T)$, for any $\rho$ satisfying $0<\rho<\tau$. Set $U(t)=\int_{B_{\gamma}(x)}\left|\tilde{\nabla}_{D}^{(n)} A\right|^{p} d V$. Then (4.21) gives

$$
\frac{d}{d t} U(t) \leq C U(t)+C
$$

Using Gronwall's inequality, we get

$$
\sup _{\tau<t<T} \int_{B_{r / 2}(x)}\left|\tilde{\nabla}_{D}^{(n)} A\right|^{p} d V \leq C .
$$

Remark that

$$
\int_{B_{\gamma / 2}(x)}\left|\nabla^{(n)} A\right|^{p} d V \leq \int_{B_{\gamma / 2}(x)}\left|\tilde{\nabla}_{D}^{(n)} A\right|^{p} d V+C \int_{B_{\gamma / 2}(x)}|A|^{p}\left|\tilde{\nabla}_{D}^{(n-1)} A\right|^{p} d V .
$$

Using Lemma 4.7 , we have by induction,

$$
\int_{B_{r / 2}(x)}\left|\nabla^{(n)} A\right|^{p} d V \leq \int_{B_{r / 2}(x)}\left|\tilde{\nabla}_{D}^{(n)} A\right|^{p} d V+C
$$

Lemma 4.9. Let $R_{0}$ be as in Proposition 2.1. There exists a constant $\varepsilon_{1}>0$ such that for any smooth solution $D$ of $(1.1)$ on $(0, T)$ with the initial value $(1.2), D^{(0)} \in$ $\mathfrak{u}^{1,2}$, any $\tau>0$, any $p \geq 2$ and any number $r \in\left(0, R_{0}\right]$, if $\varepsilon(r, x)<\varepsilon_{1}$, then we have

$$
\sup _{\tau<t<T} \int_{B_{r / 2}(x)}\left|\partial_{t} A\right|^{p} d V \leq C,
$$

where $D=d+A$ on $B_{r}(x)$ and the constant $C$ depends on $\tau, T, r, E_{0}, M$, $\left\|R_{D}(\tau)\right\|_{W^{2,2}\left(B_{r}(x)\right)}$ and $\|A(\tau)\|_{L^{p}\left(B_{r^{\prime} / 2}(x)\right)}$.

Proof. Taking the norm on both sides of (1.1), we have

Thus, we get

$$
\left|\partial_{t} A\right|^{p} \leq\left|d_{D}^{*} R_{D}\right|^{p}
$$

$$
\int_{B_{r / 2}(x)}\left|\partial_{t} A\right|^{p} d V=\int_{B_{r / 2}(x)}\left|d_{D}^{*} R_{D}\right|^{p} d V \leq \int_{B_{r / 2}(x)}\left|\tilde{\nabla}_{D} R_{D}\right|^{p} d V .
$$


Lemma 4.10. Let $R_{0}$ be as in Proposition 2.1. For any smooth solution $D$ of (1.1) on $(0, T)$ with the initial value $(1.2), D^{(0)} \in \mathfrak{u}^{1,2}$ and any number $r \in\left(0, R_{0}\right]$, we have

$$
\int_{B_{r / 2}(x)}\left|R_{D}\right|^{2}(t) d V \leq \int_{B_{r}(x)}\left|R_{D}\right|^{2}(0) d V+C r^{-2} t E_{0}
$$

where the constant $C$ depends only on $M$.

Proof. Let $\varphi$ be the function defined in the proof of Lemma 4.2. Multiplying (1.1) by $\partial_{t} A \varphi^{2}$ and integrating over $M$, we have

$$
\begin{aligned}
\int_{M}\left|\partial_{t} A\right|^{2} \varphi^{2} d V & =-\int_{M}\left\langle d_{D}^{*} R_{D}, \partial_{t} A\right\rangle \varphi^{2} d V \\
& \leq-\int_{M}\left\langle R_{D}, \partial_{t} R_{D}\right\rangle \varphi^{2} d V+C \int_{M}\left|R_{D}\right|\left|\partial_{t} A\right||\nabla \varphi| \varphi d V
\end{aligned}
$$

So we have

$$
\int_{M}\left|\partial_{t} A\right|^{2} \varphi^{2} d V+\frac{1}{2} \frac{d}{d t} \int_{M}\left|R_{D}\right|^{2} \varphi^{2} d V \leq \frac{1}{2} \int_{M}\left|\partial_{t} A\right|^{2} \varphi^{2} d V+C r^{-2} \int_{B_{r}(x)}\left|R_{D}\right|^{2} d V
$$

Integrating $(4.22)$ over $[0, t]$, we have

$$
\frac{1}{2} \int_{M}\left|R_{D}\right|^{2}(t) \varphi^{2} d V \leq \frac{1}{2} \int_{B_{r}(x)}\left|R_{D}\right|^{2}(0) d V+C r^{-2} t E_{0}
$$

which gives Lemma 4.10 .

\section{Existence of global weak solutions}

Let $T=T\left(D^{(0)}\right)$ be the maximal existence time for the smooth solution of (1.1).

Theorem 5.1. Let $D^{(0)} \in \mathfrak{u}^{1,2}$, and let $D$ be a solution of (1.1) with the initial condition (1.2) on $\left(0, T\left(D^{(0)}\right)\right)$. Then we have the following:

(1) $T\left(D^{(0)}\right)$ is characterized by

$$
\limsup _{T^{\prime}} \sup _{x \in M}\left(\int_{B_{r}(x)}\left|R_{D}\right|^{2} d V\right)^{1 / 2} \geq \varepsilon_{1} \text { for all } r \in\left(0, R_{0}\right] .
$$

(2) If $T<\infty$, then the solution $D$ is smooth on $M \times(0, T]$ except for finitely many points $\left\{\left(x^{l}, T\right): 1 \leq l \leq L\right\}$. 
(3) The singular point $\left(x^{l}, T\right)$ is characterized by

$$
\limsup _{T^{\prime} \uparrow T}\left(\int_{B_{\gamma^{\prime}}\left(x^{l}\right)}\left|R_{D}\right|^{2} d V\right)^{1 / 2} \geq \varepsilon_{1} \text {, for all } r \in\left(0, R_{0}\right] \text {. }
$$

(4) The energy $E(D(\cdot, t))$ is non-increasing.

Proof. By Theorem 3.1, there exist a local smooth solution $D(t)$ of (1.1). (See Remark 3.6). By using Lemmas 4.2-4.9, we see that the maximal existence time $T\left(D^{(0)}\right)$ is characterized by

$$
\limsup _{T^{\prime} \rightarrow T\left(D^{(0)}\right)}\left(\int_{B_{r}(x)}\left|R_{D}\right|^{2} d V\right)^{1 / 2} \geq \varepsilon_{1}
$$

for all $r>0$ and some $x \in M$. Theorem 5.1 follows immediately from:

Lemma 5.2. Put

$$
\mathscr{S}_{T^{*}}:=\left\{x \in M: \int_{B_{r}(x)}\left|R_{D}\left(\cdot, T^{*}\right)\right|^{2} d V \geq \varepsilon_{1} \quad \text { for all } r \in\left(0, R_{0}\right]\right\} .
$$

Then $\mathscr{S}_{T^{*}}$ consists of finitely many points.

The lower semi-continuity of the energy yields

$$
\begin{aligned}
\int_{M^{\prime} \times\left\{T^{*}\right\}}\left|R_{D}\right|^{2} d V & \leq \liminf _{T \rightarrow T^{*}, T<T^{*}} \int_{M^{\prime} \times\{T\}}\left|R_{D}\right|^{2} d V \\
& \leq \liminf _{T \rightarrow T^{*}, T<T^{*}} \int_{M \times\{T\}}\left|R_{D}\right|^{2} d V-\sum_{l=1}^{L_{1}} \int_{B_{r}\left(x^{l}\right) \times\left\{T^{*}\right\}}\left|R_{D}\right|^{2} d V \\
& \leq E_{0}-L_{1} \varepsilon_{1},
\end{aligned}
$$

for any $r \in\left(0, R_{0}\right]$ and any $M^{\prime} \subset M \backslash \bigcup_{l=1}^{L_{1}} B_{r}\left(x^{1}\right)$. Passing to the limit $r \rightarrow 0$, we have

$$
E\left(\cdot, T^{*}\right) \leq E_{0}-L_{1} \varepsilon_{1} .
$$

From this estimate we conclude that $L_{1}$ must be finite. This gives Lemma 5.2.

By Lemmas 4.2-4.9 and Lemma 5.2, we have the following:

THEOREM 5.3. Let $D(t)$ be a smooth solution of $(1.1)$ on $(0, T), T<\infty$. Then 
there exists a finite open covering $\left\{U_{\alpha}\right\}$ of $M \backslash\left\{x_{1}, \cdots, x_{L}\right\}$ and a connection $D$ on $P$ over $M \backslash\left\{x_{1}, \cdots, x_{L}\right\}$ satisfying the following conditions:

(1) $A_{\alpha}(t) \rightarrow A_{\alpha}$ in $W_{\text {loc }}^{n, p}\left(U_{\alpha}\right)$, for any $p \geq 2$, and any $n \geq 0$,

(2) $\left(R_{D(t)}\right)_{\alpha} \rightarrow\left(R_{D}\right)_{\alpha}$ in $W_{\text {loc }}^{n, 2}\left(U_{\alpha}\right)$, for any $n \geq 0$,

(3) $R_{D(t)} \bullet R_{D}$ weakly in $L^{2}(M)$,

as $t \uparrow T$, where $D(t)=d+A_{\alpha}(t)$ and $D=d+A_{\alpha}$ on $U_{\alpha}$.

Remark. Assume $T=\infty$, then there exists a $T_{0}>0$ such that the solution is smooth on $\left(T_{0}, \infty\right)$. By using the identity $\int_{M}\left|\partial_{t} A\right|^{2} d V=-\frac{1}{2} \frac{d}{d t} \int_{M}\left|R_{D}\right|^{2} d V$, the monotone non-increasing property of the energy, and a Sedlacek's result [8, Theorem 3.1, Theorem 4.3], there exists a finite open covering $\left\{U_{\alpha}\right\}$ of $M \backslash\left\{x_{1}, \cdots, x_{L}\right\}$, a sequence $\left\{t_{j}\right\}, t_{j} \rightarrow \infty$, gauge transformations $\left\{\sigma_{\alpha}\left(t_{j}\right)\right\}$ and a connection $D$ on $P$ over $M \backslash\left\{x_{1}, \cdots, x_{L}\right\}$ satisfying the following conditions:

(1) $\sigma_{\alpha}^{*}\left(t_{j}\right) A_{\alpha}\left(t_{j}\right) \rightarrow A_{\alpha}$ in $W_{\text {loc }}^{1,2}\left(U_{\alpha}\right)$

(2) $\sigma_{\alpha}^{*}\left(t_{j}\right) A_{\alpha}\left(t_{j}\right) \rightarrow A_{\alpha}$ in $L_{\mathrm{loc}}^{2}\left(U_{\alpha}\right)$,

(3) $R_{\sigma_{\alpha}^{*}\left(t_{j}\right) D\left(t_{j}\right)} \bullet R_{D}$ weakly in $L^{2}(M)$,

as $j \rightarrow \infty$, where $D(t)=d+A_{\alpha}(t)$ and $D=d+A_{\alpha}$ on $U_{\alpha}$. Moreover, $A_{\alpha}$ is a Yang-Mills connection on $U_{\alpha}$, hence $P$ extends to a $C^{\infty}$-bundle over $M$ and $D$ extends to a $C^{\infty}$-Yang-Mills connection in the extended bundle.

Now, we characterize the singular points for $D(t)$ :

Theorem 5.4. Let $D$ be a solution of (1.1) with the initial condition (1.2), $D^{(0)}$ $\in \mathfrak{U}^{1,2}$ constructed in Theorem 5.1 , and suppose that $\left(x_{0}, T\right), T \leq \infty$, is a singular point. Take a local coordinate $U_{\alpha}$ which contains $x_{0}$. Then there exist sequences $x_{m} \rightarrow$ $x_{0}, t_{m} \uparrow T, r_{m} \in\left(0, R_{0}\right], r_{m} \rightarrow 0$, gauge transformations $\left\{\varphi_{m}\right\}$ and a smooth Yang-Mills connection $D_{\infty}=d+A_{\infty}$ on $\mathbf{R}^{4}$ such that $\varphi_{m}^{*}\left(d+A_{r_{m},\left(x_{m}, t_{m}\right)}\right)$ tends to $D_{\infty}$ locally in $\mathfrak{u}^{2,2}$ on a local coordinate $U_{\alpha}$, where

$$
A_{\left.r_{m}, x_{m}, t_{m}\right)}(x, t):=A\left(r_{m} \cdot x+x_{m}, r_{m}^{2} \cdot t+t_{m}\right),
$$

and $D=d+A_{\alpha}$ on $U_{\alpha}$. Moreover the Yang-Mills connection $D_{\infty}$ extends to a smooth Yang-Mills connection on $S^{4}$.

Proof. Let $x_{0}$ be a singular point of $D$ at time $T$ characterized by the condition 


$$
\limsup _{T^{\prime} \rightarrow T} \int_{\beta_{r}\left(x_{0}\right)}\left|R_{D}\right|^{2} d V \geq \varepsilon_{1}
$$

and let $U_{\alpha}$ be a local coordinate of $M$ satisfying $x \in U_{\alpha}$. Moreover let $\rho \in$ $\left(0, R_{0} / 2\right]$ such that $B_{\rho}\left(x_{i}\right) \cap B_{\rho}\left(x_{j}\right)=\varnothing$ for all $i \neq j$ and for each $i$ there exists $U_{\alpha}$ such that $B_{\rho}\left(x_{i}\right) \subset U_{\alpha}$. Under the expression $D=d+A_{\alpha}$ on $U_{\alpha}$, there exist sequences $x_{m} \rightarrow x_{0}, t_{m} \rightarrow T, r_{m} \in\left(0, R_{0}\right)$ with $r_{m} \rightarrow 0$ such that

$$
\varepsilon_{1}=\int_{B_{r_{m}}\left(x_{m}\right) \times\left\{t_{m}\right)}\left|R_{D}\right|^{2} d V .
$$

By Lemma 4.2 and Lemma 4.10 , we have for any $t \in\left[t_{m}-\varepsilon r_{m}^{2}, t_{m}\right]$,

$$
\int_{B_{2 r_{m}}\left(x_{m}\right) \times\{t\}}\left|R_{D}\right|^{2} d V \geq \frac{\varepsilon_{1}}{2}, \int_{t_{m}-\varepsilon r_{m}^{2}}^{t_{m}} \int_{M}\left|\tilde{\nabla}_{D} R_{D}\right|^{2} d V d t \leq C,
$$

where $\varepsilon=\frac{\varepsilon_{1}}{2 C_{1} E_{0}}$, and $C_{1}$ is the constant in Lemma 4.10 .

Hence the sequence $A_{m}:=A_{r_{m},\left(x_{m}, t_{m}\right)}$ satisfies the estimates on $\mathscr{Q}_{m}:=\{(x, t)$ : $\left.r_{m} \cdot x+x_{m} \in B_{\rho}\left(x_{0}\right), r_{m}^{2} \cdot t+t_{m} \geq 0\right\}$ :

$$
\begin{aligned}
& \sup _{\substack{(x, t) \in \mathscr{Q}_{m} \\
-\varepsilon \leq t \leq 0}} \int_{B_{1}(0)}\left|R_{D_{m}}\right|^{2} d V \leq \varepsilon_{1} \\
& \int_{\mathscr{Q}_{m}, t \in[-\varepsilon, 0]}\left|\tilde{\nabla}_{D_{m}} R_{D_{m}}\right|^{2} d V d t=\int_{t_{m}-\varepsilon r_{m}^{2}}^{t_{m}} \int_{M}\left|\tilde{\nabla}_{D} R_{D}\right|^{2} d V d t \leq C, \\
& \int_{\mathscr{Q}_{m}, t \in[-\varepsilon, 0]}\left|\partial_{t} D_{m}\right|^{2} d V d t=\int_{t_{m}-\varepsilon r_{m}^{2}}^{t_{m}} \int_{M}\left|\partial_{t} A\right|^{2} d V d t \rightarrow 0(m \rightarrow \infty),
\end{aligned}
$$

where $D_{m}=d+A_{m}$. Especially, for some $\tau_{m} \in[-\varepsilon, 0]$, we have

$$
\begin{aligned}
& \int_{\mathcal{Q}_{m}, t=\tau_{m}}\left|\tilde{\nabla}_{D_{m}} R_{D_{m}}\right|^{2} d V=C, \\
& \int_{\mathscr{Q}_{m}, t=\tau_{m}}\left|\partial_{t} D_{m}\right|^{2} d V \rightarrow 0, \\
& \int_{B_{2}(0) \times\left\{\tau_{m}\right\}}\left|R_{D_{m}}\right|^{2} d V \geq C>0,
\end{aligned}
$$

as $m \rightarrow \infty$, uniformly in $m$. Rescaling $t_{m} \mapsto t_{m}-\tau_{m} r_{m}^{2}$, we may assume $\tau_{m}=0$.

Therefore there exist suitable gauge transformations $\left\{\varphi_{m}\right\}$ and a subsequence $\left\{D_{m}\right\}$ such that $\varphi_{m}^{*} D_{m}(\cdot, 0)$ converges to $D_{\infty}$ weakly in $\mathfrak{u}^{1, p}\left(\mathbf{R}^{4}\right)$ for any $p \geq 2$ and strongly $\mathfrak{u}_{\mathrm{loc}}^{1,2}\left(\mathbf{R}^{4}\right)$. Passing to the limit $m \rightarrow \infty$, we see that $D_{\infty}$ is a 
Yang-Mills connection with finite energy on $\mathbf{R}^{4}$. By a Uhlenbeck's result [12], $D_{\infty}$ extends to a Yang-Mills connection on a bundle $P^{\prime}$ over $S^{4}$.

By Theorem 5.3 and Theorem 5.1, there exists a solution $D(t)$ of (1.1) with the initial value (1.2), $D^{(0)} \in \mathfrak{u}^{1,2}$ and the solution converges to a connection $D^{(0)}$ over $M \backslash \mathscr{S}_{T}$ as $t \rightarrow T$, which is characterized by Theorem 5.1. Moreover the curvature form $R_{D}(t)$ of $D(t)$ weakly converges to a $R$ in $L^{2}(M)$. Following the proof of Theorem 5.4, we may assume $U_{\alpha} \cong \mathbf{R}^{4}$ and we have a Yang-Mills connection on $\mathbf{R}^{4}$. By using a conformal change of coordinates, we have a gauge change $\varphi_{\alpha}$ on $U_{\alpha}$, which pull-back of the Yang-Mills connection on $S^{4}$ to that on $U_{\alpha}$. Now, set $g_{\alpha \beta}^{\prime}=\varphi_{\alpha}=\varphi_{a} \cdot g_{\alpha \beta}$, for $U_{\alpha} \cap U_{\beta} \neq \varnothing$ and $g_{\beta \gamma}^{\prime}=g_{\beta \gamma}$ for $\beta, \gamma \neq \alpha$. Then we have a new $G$-bundle $P^{\prime}$ on $M$ with the transition functions $\left\{g_{\beta \gamma}^{\prime}\right\}$. Using the Theorem in [8, Appendix], we see that $P^{\prime}$ does not depend on the choice of $r_{m}, x_{m}, t_{m}$, because, $\eta\left(P^{\prime}\right)$ and the Pontrjagin number $p_{1}\left(P^{\prime}\right)$ do not change (see Section 7$)$.

We first study the behaviour of the first Pontrjagin number. It is known that the first Pontrjagin number of a principal $G$-bundle over a 4 -manifold with the connection $D$ is

$$
p_{1}(P)=\frac{1}{4 \pi^{2}} \int_{M}\left(\left|R_{D}^{+}\right|^{2}-\left|R_{D}^{-}\right|^{2}\right) d V,
$$

where $R_{D}^{+}$and $R_{D}^{-}$is the self-dual and anti-self-dual part of $R_{D}$, respectively. Let

$$
\begin{gathered}
p_{1}(0)=\frac{1}{4 \pi^{2}} \int_{M}\left(\left|R_{D}^{+}(0)\right|^{2}-\left|R_{D}^{-}(0)\right|^{2}\right) d V, \\
p_{1}(T)=\frac{1}{4 \pi^{2}} \int_{M}\left(\left|R_{D}^{+}(T)\right|^{2}-\left|R_{D}^{-}(T)\right|^{2}\right) d V,
\end{gathered}
$$

then $p_{1}(0)$ is the first Pontrjagin number of the bundle $P$.

\section{PROPOSITION 5.5. $\quad p_{1}(T) \in \mathbf{Z}$.}

Proof. Without loss of generality, we may assume that the singular set consists only one point, i.e., $\mathscr{\bigotimes}_{T}=\left\{x_{0}\right\}$. By the lower-semi-continuity for the $L^{2}$-norm of $R_{D}^{ \pm}$, we have

$$
\begin{aligned}
& p_{1}(T)-p_{1}(0) \\
= & \frac{1}{4 \pi^{2}}\left(\int_{M}\left(\left|R_{D}^{+}(T)\right|^{2}-\left|R_{D}^{-}(T)\right|^{2}\right) d V-\int_{M}\left(\left|R_{D}^{+}(0)\right|^{2}-\left|R_{D}^{-}(0)\right|^{2}\right) d V\right)
\end{aligned}
$$




$$
=\frac{1}{4 \pi^{2}}\left(\int_{M}\left(\left|R_{D}^{+}(T)\right|^{2}-\left|R_{D}^{+}(0)\right|^{2}\right) d V-\int_{M}\left(\left|R_{D}^{-}(T)\right|^{2}-\left|R_{D}^{-}(0)\right|^{2}\right) d V\right)
$$

By Theorem 5.4, there exist sequences $x_{m} \rightarrow x_{0}, t_{m} \uparrow T$ and $r_{m} \rightarrow 0$ such that $A_{r_{m}\left(x_{m}, t_{m}\right)}$ converges to $A_{\infty}$ in $W^{1,2}$. For the self-dual part, we have

$$
\begin{aligned}
& \int_{M}\left(\left|R_{D}^{+}(T)\right|^{2}-\left|R_{D}^{+}\left(t_{m}\right)\right|^{2}\right) d V \\
= & \int_{B_{r_{m}}(0)}\left(\left|R_{D}^{+}(T)\right|^{2}-\left|R_{D}^{+}\left(t_{m}\right)\right|^{2}\right) d V+\int_{M \backslash B_{r_{m}}(0)}\left(\left|R_{D}^{+}(T)\right|^{2}-\left|R_{D}^{+}\left(t_{m}\right)\right|^{2}\right) d V .
\end{aligned}
$$

Then, $\int_{B_{r_{m}}(0)}\left|R_{D}^{+}\left(t_{m}\right)\right|^{2} d V$ and $\int_{B_{r_{m}}(0)}\left|R_{D}^{+}(T)\right|^{2} d V$ converge to $\int_{S^{4}}\left|R_{D_{\infty}}^{+}\right|^{2} d V$ and 0 as $m \rightarrow \infty$, respectively. For the anti-self-dual part, we may obtain the similar result. Moreover, on $M \backslash \mathscr{S}_{T}, R_{D}^{+}\left(t_{m}\right)$ converges to $R_{D}^{+}(T)$ strongly in $L^{2}$. Since $p_{1}(0)=p_{1}\left(t_{m}\right)$ for all $m$, we have

$$
p_{1}(T)-p_{1}(0)=\text { the first Pontrjagin number of the bundle on which } D_{\infty} \text { lies. }
$$

Hence $p_{1}(T) \in \mathbf{Z}$.

In Section 7, we will prove that the obstruction $\eta(P)$ of the bundle $P$ does not change under the weak convergence of $R_{D}(t)$, (cf. Theorem 7.1). We may assume $\mathscr{S}_{T}$ contains only one point $x_{0}$. Take a local coordinate $U_{\alpha}$ which contains $x_{0}$ so that other coordinates $U_{\beta}, \alpha \neq \beta$ do not contain $x_{0}$. Together with the trivialization $\varphi_{\alpha}$ given in the arguments after the proof of Theorem 5.4, we consider the transition function $g_{\alpha \beta}^{\prime}=\varphi_{\alpha}^{*} g_{\alpha \beta}$ and $g_{\beta \gamma}^{\prime}=g_{\beta \gamma}$ for $\beta, \gamma \neq \alpha$, where $\left\{g_{\alpha \beta}\right\}$ is the transition function for $P$. Then $\left\{g_{\alpha \beta}^{\prime}\right\}$ gives a bundle $P^{\prime}$ over $M$. By the construction $R_{D}(t)$ given in Theorem 5.3 can be viewed as the $L^{2}$-section of $\Omega^{2}\left(\mathrm{~g}_{P}\right)$. In this section, let $\left\{g_{\alpha \beta}^{\prime}\right\}$ be a family of transition functions of $P^{\prime}$. By Theorem 5.3, there exists the connection $d+A_{\alpha}(T)$ on $U_{\alpha}$ such that $d A_{\alpha}(T)+\left[A_{\alpha}(T)\right.$, $\left.A_{\alpha}(T)\right]=R_{D_{\alpha}}(T)$ on $U_{\alpha}$ and we have $U_{\alpha} \cap \mathscr{S}_{T}=\varnothing$. By using the gauge transformation, we set

$$
A_{\alpha}=g_{\alpha \beta}^{\prime-1} d g_{\alpha \beta}^{\prime}+g_{\alpha \beta}^{\prime-1} A_{\beta} g_{\alpha \beta}^{\prime} .
$$

Also, by the construction of $P^{\prime}$, a family $\left\{A_{\beta}\right\}$ is extendable to a $W^{1,2}$-connection on $P^{\prime}$ and satisfies $R_{D^{(1)}}=R_{D}(T)$ on $M \backslash \mathscr{S}_{T}$ so that $\left|R_{D}(t)\right|$ converges to $\left|R_{D^{(1)}}\right|$ in $L^{2}(M)$.

Using the connection $D^{(1)}$, we see that the solution $D(t)$ is extendable beyond 
$T$ weakly, and as a result a weak global solution of (1.1) can be obtained.

Theorem 5.6 (existence of global weak solution). Let $M$ be a closed 4-manifold. For any initial connection $D^{(0)} \in \mathfrak{u}^{1,2}$ on $P$, there exist finite sets $\left\{t_{i}\right\}_{i=1}^{L}$, $\left\{x_{i}\right\}_{i=1}^{L} N_{i=1}$, where $N_{i}<\infty$, a finite correction of $G$-bundles $\left\{P_{i}\right\}_{i=1}^{L+1}$, where $P_{1}=P$ and a solution $D(t)$ of $(1.1)$ on $(0, \infty) \times M$ with the initial condition (1.2) such that $D(t)$ is a connection of $P_{i}$ on $\left(t_{i-1}, t_{i}\right)$ and $D\left(t_{i}\right)$ is a connection $\left.P_{i}\right|_{M \backslash \cup_{j=1}^{N_{i}}\left(x_{i}\right\}} \cong$ $\left.P_{i+1}\right|_{M \backslash \cup_{j=1}^{N}\left(x_{i}\right\}}$. Moreover, the energy $E(D(\cdot, t))$ is non-increasing.

Proof. By Theorem 3.1, we find a time local solution $D(t)$ of $(1.1)$ with the initial value $D^{(1)}$ at the time $T$. Assume the solution is smooth on $\left(T_{0}, T_{1}\right), T_{1}>$ $T_{0}$. Then the solution converges to a connection in the sense of Theorem 5.3 as $t \rightarrow T_{1}$, and we have $E\left(T_{1}\right) \leq E\left(T_{0}\right)-\varepsilon_{1} N_{*}$. Iterating this procedure, we see that the solution $D(t)$ can be extended up to $t=\infty$.

Since the energy functional $E(t)=\frac{1}{2} \int_{M}\left|R_{D}(t)\right|^{2} d V$ is monotone nonincreasing with respect to $t$ the initial condition as $E\left(D^{(0)}\right) \leq \varepsilon_{1}$ allows neither blow-up time nor local concentration of the energy. In such a situation, we get the global smooth solution of (1.1):

COROLlary 5.7 (global smooth solution with small initial energy). If $E\left(D^{(0)}\right) \leq \varepsilon_{1}$, then there exists a smooth global solution for (1.1) with the initial condition (1.2), $D^{(0)} \in \mathfrak{u}^{1,2}$.

\section{Uniqueness of solutions}

We now prove Theorem C:

Theorem 6.1. Let $D=D_{0}+\mathscr{A}$ and $\bar{D}=D_{0}+\bar{A}$ be two weak solution of (1.1) with the same initial condition (1.2) in the class of $X(M,(0, T))$. Suppose in addition that $\mathscr{A}, \bar{A} \in L^{q}\left(0, T ; L^{r}\left(\Omega^{1}\left(\mathfrak{g}_{p}\right)\right)\right)$ for $q \geq 2$ and $r>4$ with $2 / q+4 / r \leq 1$. If $d_{D_{0}}^{*} \mathscr{A}, d_{D_{0}}^{*} \bar{A} \in W^{1, \infty}\left(M \times[0, T) ; \Omega^{0}\left(\mathrm{~g}_{P}\right)\right)$, then there exist gauge transformations $s$ and $\bar{s}$ in the class $W^{1, \infty}\left(M \times[0, T) ; \mathscr{S}_{P}\right)$ such that $s^{*} \mathscr{A}=\bar{s}^{*} \bar{A}$ on $M \times[0, T)$.

Remark. (1) By the Sobolev embedding, we have an inclusion $X(M,(0, T))$ $\hookrightarrow L^{\infty}\left(0, T ; L^{4}\left(\Omega^{1}\left(\mathfrak{g}_{p}\right)\right)\right)$. Hence $X(M,(0, T))$ is a limiting case in $L^{q}(0, T$; $\left.L^{r}\left(\Omega^{1}\left(\mathfrak{g}_{p}\right)\right)\right)$ as $q \uparrow \infty$ and $r \downarrow 4$ in the relation $2 / q+4 / r \leq 1$. 
(2) For such gauge transformations $s$ and $\bar{s}$ as above, we have $s^{*} \mathscr{A}, \bar{s}^{*} \bar{A} \in$ $L^{\infty}\left(0, T ; W^{1,2}\left(\Omega^{1}\left(\mathfrak{g}_{P}\right)\right)\right) \cap L^{q}\left(0, T ; L^{r}\left(\Omega^{1}\left(\mathfrak{g}_{P}\right)\right)\right) ; s$ and $\bar{s}$ preserve regularity of $\mathscr{A}$ and $\bar{A}$, respectively.

Theorem 6.1 is proved by establishing the following lemmas.

Lemma 6.2. Let $f \in W^{1, \infty}\left(M \times[0, T] ; \Omega^{0}\left(g_{p}\right)\right)$. Then there exists a unique gauge transformation $s \in W^{1, \infty}\left(M \times[0, T] ; \mathbb{S}_{P}\right)$ such that

$$
\left\{\begin{array}{l}
s^{-1} \partial_{t} s=f \text { in } M \times(0, T), \\
s(0)=\mathrm{id} .
\end{array}\right.
$$

For the construction of the solution $s(t)$, we may use the successive approximation:

$$
\left\{\begin{array}{l}
s_{0}(t)=\mathrm{id} \\
s_{m+1}(t)=s_{0}(t)+\int_{0}^{t} s_{m}(\tau) f(\tau) d \tau, m=0,1, \cdots
\end{array}\right.
$$

Then we can easily show that $s_{m} \rightarrow s$ in $W^{1, \infty}\left(M \times[0, T] ; \Omega^{0}\left(\mathfrak{g}_{P}\right)\right)$, which yields the desired solution (see, e.g., Nagasawa [6, Theorem 3.2.1]).

Lemma 6.3. Let $A$ and $\bar{A}$ be two solutions of (3.1) in the sense of distribution in the class $L^{\infty}\left(0, T ; W^{1,2}\left(\Omega^{1}\left(\mathfrak{g}_{p}\right)\right)\right) \cap L^{r}\left(0, T ; L^{r}\left(\Omega^{1}\left(\mathfrak{g}_{p}\right)\right)\right)$, where $q \geq 2$ and $r>$ 4 with $2 / q+4 / r \leq 1$. Then we have $A=\bar{A}$ on $M \times[0, T)$.

For a moment, let us assume Lemma 6.3.

Proof of Theorem 6.1. By the assumption in Theorem 6.1, $d_{D_{0}}^{*} \mathscr{A}, d_{D_{0}}^{*} \bar{A} \in$ $W^{1, \infty}\left(M \times[0, T) ; \Omega^{0}\left(g_{P}\right)\right)$ and it follows from Lemma 6.2 that there exist gauge transformations $s$ such that

$$
\left\{\begin{array}{l}
s^{-1} \partial_{t} s=d_{D_{0}}^{*} \mathscr{A}, \bar{s}^{-1} \partial_{t} \bar{s}=d_{D_{0}}^{*} \bar{A}, t>0 \\
s(0)=\bar{s}(0)=\mathrm{id}
\end{array}\right.
$$

Defining $A=s^{*} \mathscr{A}$ and $\bar{A}=\bar{s}^{*} \bar{A}$, we obtain $A, \bar{A} \in L^{\infty}\left(0, T ; W^{1,2}\left(\Omega^{1}\left(\mathfrak{g}_{P}\right)\right)\right) \cap$ $L^{q}\left(0, T ; L^{r}\left(\Omega^{1}\left(g_{P}\right)\right)\right.$ ) (see Uhlenbeck [13, Lemma 1.2]). Moreover, the derivation of (3.1) enables us to see that $A$ and $\bar{A}$ are weak solutions of (3.1) with the same initial data. So, Lemma 6.2 yields the desired result. 
Now it remains to prove Lemma 6.3.

Proof of Lemma 6.3. By the Sobolev embedding, we have

$$
\begin{aligned}
|\langle Q(A), \varphi\rangle| & \leq\left|\left\langle Q_{1}(A), \varphi\right\rangle\right|+\left|\left\langle Q_{2}(A), \varphi\right\rangle\right| \\
& \leq C\left(\|A\|_{4}\|\nabla A\|_{2}\|\varphi\|_{4}+\|A\|_{4}^{3}\|\varphi\|_{4}\right) \\
& \leq C\left(\|A\|_{W^{1,2}}^{2}+\|A\|_{W^{1,2}}^{3}\right)\|\varphi\|_{W^{1,2}}
\end{aligned}
$$

for all $\varphi \in W^{1,2}\left(\Omega^{1}\left(\mathrm{~g}_{p}\right)\right)$, from which

$$
Q(A), Q(\bar{A}) \in L^{\infty}\left(0, T ; W^{1,2}\left(\Omega^{1}\left(g_{P}\right)\right)^{*}\right) .
$$

Hence $A$ and $\bar{A}$ satisfies (3.2) in $W^{1,2}\left(\Omega^{1}\left(\mathfrak{g}_{p}\right)\right)^{*},\left(Y^{*}\right.$ : dual space of $\left.Y\right)$.

Taking $B=A-\bar{A}$, we have $B \in C^{0}\left([0, T) ; L^{2}\left(\Omega^{1}\left(\mathfrak{g}_{P}\right)\right)\right)$ and

$$
\left\{\begin{array}{l}
\frac{\partial B}{\partial t}+\mathscr{L} B+Q(A)-Q(\bar{A})=0 \text { in } W^{1,2}\left(\Omega^{1}\left(\mathfrak{g}_{P}\right)\right)^{*}, \\
B(0)=0 .
\end{array}\right.
$$

Since $Q(A), Q(\bar{A}) \in L^{\infty}\left(0, T ; W^{1,2}\left(\Omega^{1}\left(g_{P}\right)\right)^{*}\right)$, it follows from the definition of the weak solution that $\frac{\partial B}{\partial t} \in L^{\infty}\left(0, T ; W^{1,2}\left(\Omega^{1}\left(\mathrm{~g}_{P}\right)\right)^{*}\right)$. Then applying Temam [11, Chapter III, Lemma 1.2], we have the identity

$$
\left\langle\frac{\partial B}{\partial t}+\mathscr{L} B, B\right\rangle=\frac{1}{2} \frac{d}{d t}\|B\|_{2}^{2}+\|\nabla B\|_{2}^{2}-\langle[R, B], B\rangle,
$$

where $\langle\cdot, \cdot\rangle$ denotes the duality pairing between $W^{1,2}\left(\Omega^{1}\left(\mathfrak{g}_{P}\right)\right)^{*}$ and $W^{1,2}\left(\Omega^{1}\left(g_{P}\right)\right)$ and $R$ is the curvature form of $D_{0}$. Using Gagliardo-Nirenberg's inequality

$$
\|B\|_{\frac{2 r}{r-2}} \leq C\|B\|_{2}^{1-4 / r}\|B\|_{W^{1,2}}^{4 / r}
$$

we obtain from Young's inequality

$$
\begin{aligned}
\left|\left\langle Q_{1}(A)-Q_{1}(\bar{A}), B\right\rangle\right| & \leq\left(\|A\|_{r}+\|\bar{A}\|_{r}\right)\|\nabla B\|_{2}\|B\|_{\frac{2 r}{r-2}} \\
& \leq C\left(\|A\|_{r}+\|\bar{A}\|_{r}\right)\|B\|_{W^{1,2}}^{1+4 / r}\|B\|_{2}^{1-4 / r} \\
& \leq \frac{1}{4}\|\nabla B\|_{2}^{2}+C\left(1+\|A\|_{r}^{\frac{2 r}{r-4}}+\|\bar{A}\|_{r}^{\frac{2 r}{r-4}}\right)\|B\|_{2}^{2}, \\
\left|\left\langle Q_{2}(A)-Q_{2}(\bar{A}), B\right\rangle\right| & \leq\left(\|A\|_{r}^{2}+\|\bar{A}\|_{r}^{2}\right)\|B\|_{r r}^{2} \\
& \leq C\left(\|A\|_{r}^{2}+\|\bar{A}\|_{r}^{2}\right)\|B\|_{W^{1,2}}^{8 / r}\|B\|_{2}^{2(1-4 / r)}
\end{aligned}
$$




$$
\leq \frac{1}{4}\|\nabla B\|_{2}^{2}+C\left(1+\|A\|_{r}^{\frac{2 r}{r-4}}+\|\bar{A}\|_{r}^{\frac{2 r}{r-4}}\right)\|B\|_{2}^{2} .
$$

Now it follows from (6.1)-(6.4)

$$
\frac{d}{d t}\|B\|_{2}^{2}+\|\nabla B\|_{2}^{2} \leq C\left(1+\|R\|_{\infty}+\|A\|_{r}^{\frac{2 r}{r-4}}+\|\bar{A}\|_{r}^{\frac{2 r}{r-4}}\right)\|B\|_{2}^{2} .
$$

Since $\frac{2 r}{r-4} \leq q$, we have by assumption $\int_{0}^{T}\|A(t)\|_{r}^{\frac{2 r}{r-4}} d t, \int_{0}^{T}\|\bar{A}(t)\|_{r}^{\frac{2 r}{r-4}} d t$ $<\infty$ and hence (6.5) and Gronwall's inequality yield the conclusion. This completes the proof of Lemma 6.3.

\section{Topology of bundles}

In this section, we study structures of bundles on which defined the weak solution $D(t)$. In Section 5 , we have proved the behaviour of the first Pontrjagin number $p_{1}$, so we will study the obstruction $\eta(P)$. The idea in this section is due to Sedlack [8].

Note that $p_{1}$ may not be conserved in time, however, we can find a conservation quantity in time.

THEOREM 7.1. For the weak solution $D(t)$ of (1.1) with the initial condition (1.2), $D^{(0)} \in \mathfrak{u}^{1,2}$, the obstruction $\eta(P)$ is conserved in all time.

Proof. Let $T>0$ be the first singular time of the solution $D(t)$. Since $D(t)$ is smooth on $0<t<T$, there exist a family of transition functions $\left\{g_{\alpha \beta}\right\}$ of the bundle $P$ such that $A_{\beta}(t)=g_{\alpha \beta}(t)^{-1} d g_{\alpha \beta}(t)+g_{\alpha \beta}(t)^{-1} A_{\alpha}(t) g_{\alpha \beta}(t)$. First, we observe that $\left\{g_{\alpha \beta}(t)\right\}$ are $W^{1,4}$-bounded on $U_{\alpha} \cap U_{\beta}$ for $t \rightarrow T$. On $U_{\alpha} \cap U_{\beta}$ we have

$$
\begin{aligned}
\left\|d g_{\alpha \beta}(t)\right\|_{L^{4}\left(U_{\alpha} \cap U_{\beta}\right)} & \leq\left\|A_{\beta}(t)\right\|_{L^{4}\left(U_{\alpha} \cap U_{\beta}\right)}+\left\|A_{\alpha}(t)\right\|_{L^{4}\left(U_{\alpha} \cap U_{\beta}\right)} \\
& \leq C\left(\left\|A_{\beta}(t)\right\|_{W^{1,2}\left(U_{\alpha} \cap U_{\beta}\right)}+\left\|A_{\alpha}(t)\right\|_{W^{1,2}\left(U_{\alpha} \cap U_{\beta}\right)}\right) .
\end{aligned}
$$

Note that the right hand side of (7.1) is bounded on $t \in(0, T]$, if $U_{\alpha} \cap \mathscr{S}_{T}=\varnothing$ and $U_{\beta} \cap \mathscr{S}_{T}=\varnothing$. Therefore we conclude the $W^{1,4}$-boundedness on $U_{\alpha} \cap U_{\beta}$ of $\left\{g_{\alpha \beta}(t)\right\}$.

Since the projection map $\pi$ is an isometry, we have

$$
\left\|d \tilde{g}_{\alpha \beta}(t)\right\|_{L^{4}\left(U_{\alpha} \cap U_{\beta}\right)}=\left\|\pi d \tilde{g}_{\alpha \beta}(t)\right\|_{L^{4}\left(U_{\alpha} \cap U_{\beta}\right)}=\left\|d g_{\alpha \beta}(t)\right\|_{L^{4}\left(U_{\alpha} \cap U_{\beta}\right)},
$$


therefore $\left\{\tilde{g}_{\alpha \beta}(t)\right\}$ is $W^{1,4}$-bounded on $U_{\alpha} \cap U_{\beta}$.

On the other hand, we can find following results in [8, Section 5].

Lemma 7.2. Let $\&=\left\{x_{1}, \cdots, x_{N}\right\}$ be a set of finitely many points in $M$, and let $J: M \backslash \& \rightarrow M$ be the inclusion map. For principal $G$-bundles $P$ and $P^{\prime}$ over $M$, if $\eta\left(J^{*} P\right)=\eta\left(J^{*} P^{\prime}\right)$, then $\eta(P)=\eta\left(P^{\prime}\right)$.

LEMMA 7.3. Let $f: U_{\alpha} \rightarrow \mathbf{R}$ be a finitely many valued function. If $f \in W^{1,1}$, then $f$ must be constant function.

Lemma 7.4. If the lift $\tilde{g}_{\alpha \beta}$ of $g_{\alpha \beta} \in C^{\infty}$ is $W^{1,4}$, then $\tilde{g}_{\alpha \beta} \in C^{\infty}$.

Let $\mathscr{S}=\left\{x_{i}\right\}$ be the singular points of the weak solution at $t=T$. By Lemma 7.2. it is sufficient to prove on $M \backslash \&$. The functions $f_{\alpha \beta \gamma}(t)(x)=\tilde{g}_{\alpha \beta}(t)(x)$. $\tilde{g}_{\beta \gamma}(t)(x) \cdot \tilde{g}_{\gamma \alpha}(t)(x)$ converges to $\tilde{g}_{\alpha \beta}(T)(x) \cdot \tilde{g}_{\beta \gamma}(T)(x) \cdot \tilde{g}_{\gamma \alpha}(T)(x)=f_{\alpha \beta \gamma}(T)(x)$ for any $x \in M \backslash \mathscr{S}$ in $C^{\infty}$, by Lemma 7.4 and Lemma 7.3. Therefore, we have $\eta(P)=\eta\left(P^{\prime}\right)$

Remark. The obstruction $\eta(P)$ coincides the second Stiefel-Whitney class $w_{2}(P) \in H^{2}\left(M, \mathbf{Z}_{2}\right)$, if $G=O(n)$ or $S O(n)$. If $G=U(n), \eta(P)$ coincides the first Chern class $c_{1}(P) \in H^{2}(M, \mathbf{Z})$.

Remark. By following the argument in Sedlacek [8], the assumption that $\tilde{G}$ is compact will be removed.

\section{Acknowledgement}

The authors would like to thank Professor Hiraku Nakajima and Professor Shigetoshi Bando for valuable comments. Finally, we would like to express our hearty thanks to the referee for his/her valuable comments.

The second author would like to thank the Erwin Schrödinger International Institute for Mathematical Physics for their hospitality.

Note added in Proof. After the completion of the present paper, Professor M. Struwe kindly informed us his recent work [Cacl. Var. 2 (1994), 123-150], in which they independently had shown similar results. However, our method is different from his method. 


\section{REFERENCES}

[1] D. Fujiwara, On the asymptotic behaviour of the Green operators for elliptic boundary problems and the pure imaginary powers of the second order operators, J. Math. Soc. Japan, 21 (1969), 481-521.

[2] J. Jost, "Nonlinear Methods in Riemannian and Kählerian Geometry," Birkhäuser, Basel, 1988.

[ 3 ] K. Kono and T. Nagasawa, Weak asymptotic stability of Yang-Mills gradient flow, Tokyo J. Math., 11 (1988), 339-357.

[4] O. A. Ladyženskaja, V. A. Solonnikov and N. N. Ural'ceva, "Linear and Quasilinear Equations of Parabolic Type," American Mathematical Society, Providence, 1968.

[5] J. Moser, A Harnack inequality for parabolic differential equations, Commun. Pure and Apply. Math., 17 (1964), 101-134.

[6] T. Nagasawa, Asymptotic stability of Yang-Mills' gradient flow, in "Geometry of Manifolds (edited by K. Shiohama)," Academic Press, Boston, 1989, pp. 499-517.

[ 7 ] H. Naito, H. Kozono and Y. Maeda, A stable manifold theorem for the Yang-Mills gradient flow, Tôhoku Math. J., 42 (1990), 45-66.

[8] S. Sedlacek, A direct method for minimizing the Yang-Mills functional over 4 -manifolds, Commun. Math. Phys., 86 (1982), 515-527.

[9] M. Struwe, On the evolution of harmonic mappings of Riemann surfaces, Comm. Math. Helv., 4 (1985), 558-581.

[10] H. Tanabe, "Equation of Evolution," Pitmann, London, 1979.

[11] R. Temam, "Naviar-Stokes equations, theory and numerical analysis," NorthHolland, Amsterdam-New York- Oxford, 1979.

[12] K. Uhlenbeck, Removable singularities in Yang-Mills fields, Commun. Math. Phys., 83 (1982), 11-29.

[13] K. Uhlenbeck, Connections with $L^{p}$-bounds on curvature, Commun. Math. Phys., 83 (1982), 31-42.

Hideo Kozono

Graduate School of Polymathematics

Nagoya University

Nagoya, 464-01, Japan

Yoshiaki Maeda

Department of Mathematics

Faculty of Science and Technology

and The Erwin Schrödinger International

Keio University Institute for Mathematical Physics

Yokohama, 223, Japan

Wien, A-1090, Austria

Hisashi Naito

Graduate School of Polymathematics

Nagoya University

Nagoya, 464-01, Japan 